\title{
A solenoidal electron spectrometer for a precision measurement of the neutron $\beta$-asymmetry with ultracold neutrons
}

\author{
B. Plaster, ${ }^{\mathrm{a}, \mathrm{b}}$ R. Carr,${ }^{\mathrm{a}}$ B. W. Filippone, ${ }^{\mathrm{a}}$ D. Harrison, ${ }^{\mathrm{c}}$ \\ J. Hsiao, ${ }^{\text {a }}$ T. M. Ito, ${ }^{\text {a,d }}$ J. Liu, ${ }^{\text {a }}$ J. W. Martin,,${ }^{\text {a,c }}$ B. Tipton, ${ }^{\text {a }}$ \\ J. Yuan ${ }^{\mathrm{a}}$ \\ ${ }^{\text {a } W . ~ K . ~ K e l l o g g ~ R a d i a t i o n ~ L a b o r a t o r y, ~ C a l i f o r n i a ~ I n s t i t u t e ~ o f ~ T e c h n o l o g y, ~}$ \\ Pasadena, CA 91125, USA \\ ${ }^{\mathrm{b}}$ Department of Physics and Astronomy, University of Kentucky, \\ Lexington, $K Y$ 40506, USA \\ ${ }^{\mathrm{c}}$ Physics Department, University of Winnipeg, Manitoba, Canada R3B 2E9 \\ ${ }^{\mathrm{d}}$ Los Alamos National Laboratory, Los Alamos, NM 87545, USA
}

\begin{abstract}
We describe an electron spectrometer designed for a precision measurement of the neutron $\beta$-asymmetry with spin-polarized ultracold neutrons. The spectrometer consists of a 1.0-Tesla solenoidal field with two identical multiwire proportional chamber and plastic scintillator electron detector packages situated within 0.6-Tesla fieldexpansion regions. Select results from performance studies of the spectrometer with calibration sources are reported.
\end{abstract}

Key words: neutron $\beta$-decay, low-energy electron magnetic spectrometer, ultracold neutrons

PACS: 29.30.Aj, 29.40.Cs, 29.40.Mc, 23.20.En

\section{Introduction}

Nuclear $\beta$-decay studies have contributed significantly to our understanding of the charged-current weak interaction and, more generally, the fundamental symmetries underlying the Standard Model of electroweak interactions. Today, a rich program of $\beta$-decay studies of nuclei, free neutrons, and pions continues in earnest, with recent high-precision results providing important information on Standard Model parameters and constraints on proposed beyond-the-Standard-Model new physics scenarios [1,2,3]. 
In neutron $\beta$-decay, measurements of angular correlation parameters and the lifetime continue to probe the limits of the vector-axial-vector $(V-A)$ description of the weak interaction. Assuming the validity of the $V-A$ theory, the differential decay rate distribution of the electron and neutrino momenta and the electron energy in spin-polarized neutron $\beta$-decay (averaged over the finalstate electron spin) can be written in terms of angular correlation parameters as [4]

$$
\frac{d W}{d E_{e} d \Omega_{e} d \Omega_{\nu}} \propto N\left(E_{e}\right)\left[1+a \frac{\vec{p}_{e} \cdot \vec{p}_{\nu}}{E_{e} E_{\nu}}+\left\langle\vec{\sigma}_{n}\right\rangle \cdot\left(A \frac{\vec{p}_{e}}{E_{e}}+B \frac{\vec{p}_{\nu}}{E_{\nu}}\right)\right],
$$

where $E_{e}\left(E_{\nu}\right)$ and $\vec{p}_{e}\left(\vec{p}_{\nu}\right)$ denote, respectively, the electron (neutrino) energy and momentum; $E_{0}(=782 \mathrm{keV})$ denotes the electron endpoint energy; $N\left(E_{e}\right)=p_{e} E_{e}\left(E_{0}-E_{e}\right)^{2}$ is the electron energy spectrum; $m_{e}$ is the electron mass; and $\left\langle\vec{\sigma}_{n}\right\rangle$ denotes the neutron polarization. Neglecting recoil-order corrections, the correlation parameters $a$ (the $e-\bar{\nu}_{e}$-asymmetry), $A$ (the $\beta$ asymmetry), and $B$ (the $\bar{\nu}_{e}$-asymmetry) are functions only of $\lambda \equiv g_{A} / g_{V}$, defined as the ratio of the weak axial-vector, $g_{A}$, and vector, $g_{V}$, couplings of the nucleon to the $W$-boson [5,6]. Therefore, measurements of $a, A$, and $B$ determine $\lambda$, by itself, a fundamental parameter of the weak interaction. In addition, a value for $\lambda$, combined with a value for the lifetime, $\tau_{n}$, where [7]

$$
\frac{1}{\tau_{n}}=\frac{G_{\mu}^{2}\left|V_{u d}\right|^{2}}{2 \pi^{3}} m_{e}^{5}\left(1+3 \lambda^{2}\right)(1+\mathrm{RC}) f
$$

permits an extraction of the CKM quark-mixing matrix element $V_{u d}$, given a value for the Fermi constant $G_{\mu}$ as determined in muon decay. Here, $f$ is a phase-space factor, and $\mathrm{RC}$ represents the total effect of all electroweak radiative corrections relative to muon decay [7]. A value for $V_{u d}$ derived solely from neutron $\beta$-decay observables is particularly compelling for a first-row CKM unitarity test [8], because the result is insensitive to corrections for nuclear structure that must be applied to extractions of $V_{u d}$ from measurements of $f t$ values in superallowed $0^{+} \rightarrow 0^{+}$nuclear $\beta$-decay transitions [9].

In the remainder of this paper, we describe a solenoidal electron spectrometer designed to extract the neutron $\beta$-asymmetry parameter $A$ from measurements of the angular correlation between the neutron spin and the decay electron's momentum in the $\beta$-decay of spin-polarized ultracold neutrons. This experiment, the UCNA experiment [10] at the Los Alamos National Laboratory, will yield the first-ever measurement of $A$ with ultracold neutrons (UCN), offering different systematics than previous and ongoing measurements employing beams of polarized cold neutrons [11. The ultimate goal of the UCNA experiment is a $0.2 \%$ measurement of $A$. We begin, in Section 2 , by detailing the requirements on the spectrometer imposed by the consideration of systematic 


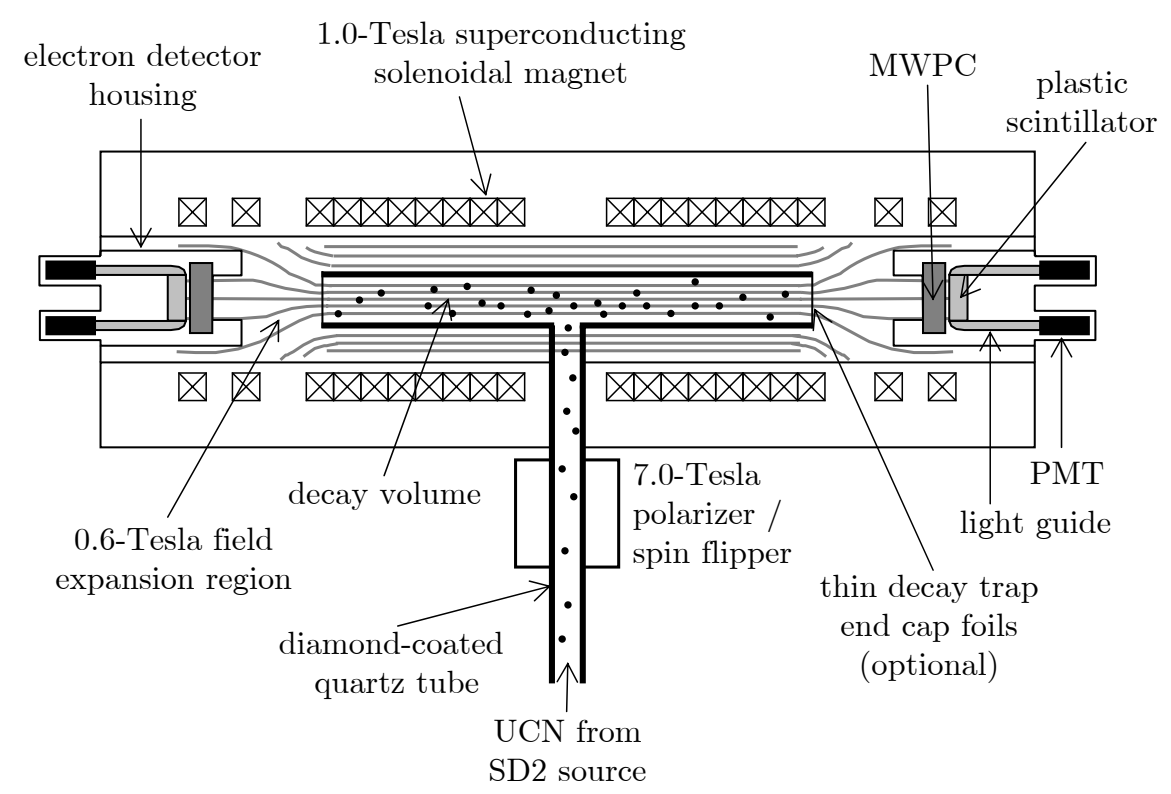

Fig. 1. Schematic diagram of the UCNA $\beta$-asymmetry experiment.

uncertainties inherent to a measurement of $A$. We follow this in Section 3 with a discussion of the spectrometer design and construction. Results from performance studies of the spectrometer with conversion-electron sources are presented in Section 4. We then conclude with a brief summary in Section 5 ,

\section{Spectrometer requirements}

\subsection{Overview of experiment}

To provide some context for a discussion of the spectrometer requirements, a schematic diagram of the UCNA experiment is shown in Fig. 1. UCN are produced in a superthermal solid deuterium source [13]14], spin-polarized via transport through a 7-Tesla field, and then directed to the center of a decay trap, where a fraction of them decay. The decay trap consists of a 3-m long, 10-cm diameter, diamond-coated quartz tube situated within the warm bore of the solenoidal spectrometer. Note that as an option to increase the UCN density in the spectrometer (and, hence, the $\beta$-decay rate), the ends of the decay trap can be closed off with thin $(<1 \mu \mathrm{m})$ foils. The spectrometer field strength of 1.0-Tesla is oriented along the axis of the decay trap (defining the spin direction) and generated by a superconducting solenoidal magnet. Two identical multiwire proportional chambers [12] and plastic scintillator detector packages are mounted on both ends of the spectrometer and situated within 0.6-Tesla field-expansion regions.

Depending on their direction of emission, the decay electrons spiral along 
the solenoidal field lines towards one of the two electron detector packages, distributed over energy and angle according to

$$
d W(\beta, \theta) \propto 1+P_{n} A \beta \cos \theta,
$$

with $P_{n}$ the neutron polarization, $\beta$ the electron velocity relative to $c$, and $\theta$ the angle of emission relative to the neutron spin (or magnetic field). The $\beta$-asymmetry $A$ is then, in principle, extracted from the (energy-dependent) experimental-asymmetry, $A_{\exp }\left(E_{e}\right)$, measured in the two detectors' count rates,

$$
A_{\exp }\left(E_{e}\right)=\frac{N_{1}\left(E_{e}\right)-N_{2}\left(E_{e}\right)}{N_{1}\left(E_{e}\right)+N_{2}\left(E_{e}\right)}=P_{n} A \beta\langle\cos \theta\rangle .
$$

The expected magnitude of the asymmetry is $A_{\exp } \sim 4.5 \%$ for $P_{n}=1$, $\langle\cos \theta\rangle=1 / 2$, and $\beta \sim 0.75$ (for $E_{e} \sim 300 \mathrm{keV}$, near the peak of the $\beta$ decay spectrum). In practice, ratios of the two detectors' count rates for neutrons polarized parallel and anti-parallel to the field [via transport through an adiabatic-fast-passage (AFP) spin-flipper field region] are formed to extract the asymmetry via a "super-ratio" technique. Here, the experimental asymmetry, $A_{\exp }\left(E_{e}\right)$, is extracted from the super ratio, $R$, according to

$$
\begin{aligned}
R & =\frac{N_{1}^{\downarrow}\left(E_{e}\right) \cdot N_{2}^{\uparrow}\left(E_{e}\right)}{N_{1}^{\uparrow}\left(E_{e}\right) \cdot N_{2}^{\downarrow}\left(E_{e}\right)}, \\
A_{\exp }\left(E_{e}\right) & =\frac{1-\sqrt{R}}{1+\sqrt{R}}=P_{n} A \beta\langle\cos \theta\rangle,
\end{aligned}
$$

where $\uparrow$ and $\downarrow$ denote the two neutron spin states. The advantage of the superratio technique is that differences between the two detectors' efficiencies and variations in the UCN density for the two spin states cancel in the ratio.

\subsection{Field uniformity requirement}

For a charged particle in a magnetic field $B$, the magnetic flux enclosed by the particle's orbit (i.e., $B r_{L}^{2}$, with $r_{L}$ the Larmor radius) is an adiabatic invariant [15]. From this, it can be shown that $p_{\perp}^{2} / B$, with $p_{\perp}$ the transverse momentum, is also an adiabatic invariant. The angle of emission relative to the magnetic field (or spin) direction is, of course, simply $\theta=\cos ^{-1}\left(p_{\|} / p\right)$, with $p_{\|}$the longitudinal momentum, and $p=\sqrt{p_{\perp}^{2}+p_{\|}^{2}}$ the total momentum. Hereafter, we will frequently refer to $\theta$ as the pitch angle for the trajectory.

Two considerations which follow from the adiabatic invariant influence the requirement on the field uniformity. First, electrons emitted with momentum 
$p_{0}=\sqrt{p_{\perp, 0}^{2}+p_{\|, 0}^{2}}$ in some local field $B_{0}$ will be reflected from field regions $B$ if $B>B_{\text {crit }} \equiv\left(p_{0}^{2} / p_{\perp, 0}^{2}\right) B_{0}$, thus contributing to a false asymmetry. Second, by this same process, electrons emitted at large pitch angles in the vicinity of a local field minimum will be trapped. These trapped electrons will scatter from residual gas isotropically and dilute the extracted value of $A$. A detailed analysis of both of these considerations showed that the effect on the asymmetry is negligible for a field uniformity of $\pm 5 \times 10^{-4}[10]$.

\subsection{Fiducial volume definition}

Unlike previous measurements of $A$ utilizing flight paths of cold neutron beams from research reactors, the UCNA experiment employs a novel technique where the polarized UCN are transported to the center of a materialwalled decay trap situated within the warm bore of the electron spectrometer. Consequently, $\beta$-decay electrons originating near the walls of the decay trap can scatter from the walls prior to impacting the electron detector packages, leading to a distortion in the measured energy spectrum. Thus, one of the requirements for the electron spectrometer is the ability to reconstruct the transverse $(x, y)$ position of the $\beta$-decay event with resolution sufficient to define a fiducial volume with a minimal loss of counting statistics.

Reconstruction of the $(x, y)$ position is achieved via readout of the induced charge on the two cathode planes of the multiwire proportional chamber (MWPC), oriented at right angles relative to each other. Complete details concerning the MWPC's design, construction, and initial offline performance tests may be found in [12. With the maximum Larmor diameter for an endpointenergy electron (with $90^{\circ}$ pitch angle) of $7.76 \mathrm{~mm}$ in the 1-Tesla field, allowing for a (conservative) $10 \%$ safety factor yields a fiducial volume radius of $(50-7.76 \times 1.10) \mathrm{mm}=41.5 \mathrm{~mm}$ (or, a $69 \%$ fiducial volume). As discussed in detail later in this paper, position resolutions of $\sim 2 \mathrm{~mm}$ have been achieved. This resolution permits identification of the $8.5 \mathrm{~mm}$ radial cut annulus with high confidence.

\subsection{Suppression of backscattering events}

Identification of electron events that undergo Coulomb backscattering from any of the MWPC components or the plastic scintillator is important for an extraction of the $\beta$-asymmetry $A[16,17]$. Particularly problematic are those events termed "missed backscattering events", in which the electron backscatters from one detector package with energy deposition below that detector's threshold. Such unidentifiable backscattering events contribute to a systematic 
shift in the extracted value of the asymmetry, and can only be corrected for in simulation. Thus, the spectrometer includes the following features designed to suppress the total and missed backscattering fractions. First, the backscattering probability increases rapidly with the angle away from normal incidence. Large pitch-angles are eliminated with the field-expansion ratio of 0.6 , which maps pitch angles of $90^{\circ}$ in the 1.0-Tesla region to $51^{\circ}$ in the 0.6 -Tesla region 1 Second, the placement of a low-energy-threshold MWPC in front of the plastic scintillator detector facilitates the identification of low-energy-deposition backscattering events which would otherwise not trigger the plastic scintillator (e.g., due to dead-layer 2 and/or threshold effects). Finally, for fixed energy, the backscattering probability increases with atomic number $Z$ [18]. Thus, the MWPCs were constructed with low- $Z$ materials [12].

Note that results from backscattering studies carried out with conversionelectron sources are not reported in this paper. Instead, a detailed companion paper comparing these data with Monte Carlo studies is in preparation [19].

\subsection{Energy reconstruction}

A measurement of the total electron energy is required, as the experimental asymmetry, $A_{\exp }\left(E_{e}\right)$ in Eq. (4), is proportional to $\beta=v / c$. The chosen detector, plastic scintillator, offers a low average atomic number $Z$, important for the suppression of backscattering events, and fast timing, defining the event trigger. The primary requirements for the scintillator detector are as follows. First, the scintillator must be sufficiently thick to provide a total energy measurement of the entire neutron $\beta$-decay spectrum up to the endpoint $E_{0}$. Second, high energy resolution is required, demanding a high photoelectron collection efficiency in the spectrometer's magnetic field environment. Third, a low energy threshold is required, again demanding a high photoelectron yield, and also a low dark rate. Fourth, the scintillator's fast timing response, coupled to fast photomultiplier tubes, permits the identification and separation of backscattering events triggering both scintillators. Fifth, the size of the scintillator is mechanically constrained by the size of the solenoidal spectrometer's warm bore. Finally, the scintillator resides in the 0.6-Tesla field expansion region, requiring that the light be transported along light guides to a lower field region ( $\sim 300$ Gauss) for readout with the photomultiplier tubes.

1 For the same reason, the transverse $(x, y)$ position of the trajectory in the 1.0Tesla region maps to that in the 0.6 -Tesla region via a $1 / \sqrt{0.6}$ factor.

2 The scintillator's dead-layer was measured offline with a variable energy 0$130 \mathrm{keV}$ electron gun and found to be $3.0 \pm 0.3 \mu \mathrm{m}[20]$. 


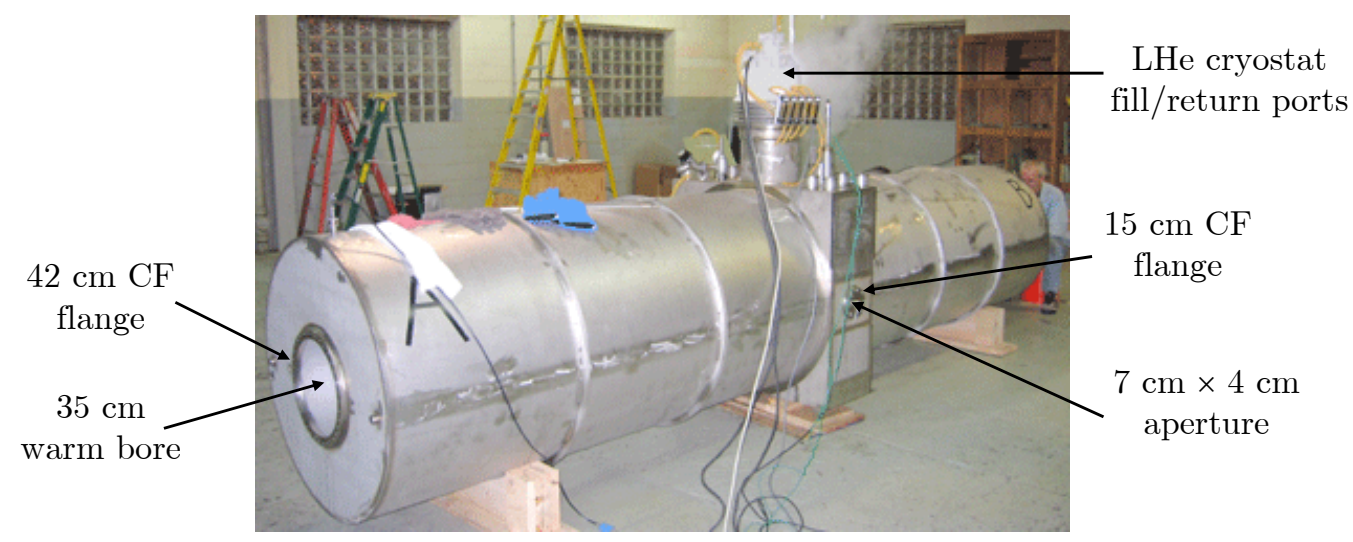

Fig. 2. (Color online) Photograph of the 1-Tesla superconducting solenoidal magnet. Provided courtesy of American Magnetics, Inc.

\subsection{Suppression of backgrounds}

One primary advantage of a neutron $\beta$-asymmetry measurement with ultracold neutrons produced via a low-frequency (typically, 1/10 to 1/20 Hz) pulsed spallation-driven superthermal solid deuterium source is the supression of (or, near absence of) beam-related backgrounds. Signal-to-noise ratios in previous $A$ experiments with cold neutron beams from research reactors were typically 7:1 [11], with gamma-ray induced events constituting the primary background. The desire for a high signal-to-noise experiment was one motivation for the introduction of the MWPC, as the requirement of a coincidence between the MWPC and the scintillator leads to a significant reduction in gamma-ray backgrounds. Further, as discussed below, the scintillator is coupled adiabatically to a series of light guides which transport the light to four photomultiplier tubes. Requiring at least a two-fold photomultiplier tube coincidence further suppresses noise from dark currents and low-energy gamma backgrounds.

\section{Spectrometer design, construction, and operation}

\subsection{1-Tesla superconducting solenoidal magnet}

A photograph of the as-built 1-Tesla superconducting solenoidal magnet is shown in Fig. 2. In general terms, the magnet is a warm-bore (35-cm diameter) 4.5-m long superconducting solenoid with three 7 -cm (vertical) $\times 4$-cm (horizontal) rectangular radial penetrations through the center of the coil. One such penetration is required for the transport of UCN to the decay trap (as indicated schematically in Fig. 1). The capacity of the magnet's liquid helium cryostat is $\sim 1600$ liters, with a heat load during operation in persistence mode equivalent to a boil-off rate of $\sim 10$ liters/hour. 
The required radial penetration complicated the design of the solenoid. Analytic studies indicated that a single split-coil solenoid would not satisfy the $\pm 5 \times 10^{-4}$ field homogeneity requirement, but a multi-coil design with varied currents/windings could be tuned to satisfy this requirement [20]. The design proposed to the chosen vendor, American Magnetics, Inc. (AMI) [21, consisted of a configuration of $3250-\mathrm{cm}$ diameter, 7 -cm wide coils (each modeled as 70 current filaments spaced $1 \mathrm{~mm}$ apart) all separated by 7 -cm, with six different currents ranging from 1600-1800 A-turns [20]. Ultimately, the designs of the coil configurations for both the 1-Tesla region and the 0.6-Tesla field-expansion region were proprietary. The vendor's end-product consisted of a main coil winding with a single persistence heater switch, 28 shim coil windings (each with individual persistence heater switches), and three rectangular 7 -cm $\times 4$-cm radial penetrations (two providing horizontal access, one providing vertical access, to the warm bore).

The cryostat for the magnet was fabricated by Meyer Tool and Manufacturing, Inc. [22]. Although the cryostat was equipped with a liquid nitrogen jacket buffer volume, in practice we have forced the (colder) liquid helium boil-off through the liquid nitrogen volume, as we have found this reduces the rate of liquid helium boil-off. Liquid helium is supplied by a helium liquefaction plant operated solely for the UCNA experiment in a closed-loop mode. This plant consists of a Koch Model 1630 liquefier coupled to a 4000-liter supply dewar, low-pressure ( $\sim$ few psi) magnet boil-off return lines coupled to 240 -psi (supply-side) compressors, and a 240-psi "medium pressure storage" ambient temperature ballast tank with $\mathrm{a} \sim 2300$ liquid liters equivalent volume.

The power supply for the main coil, an AMI Model 12200PS-420 (200 A/12 V), functions as a slave to an AMI Model 420 Power Supply Programmer unit. The full energized field strength of 1.0 Tesla requires a current of $124.0 \mathrm{~A}$. The power supply for the shim coils, a bipolar Kepco Model BOP 20-10M $( \pm 20$ $\mathrm{V} / \pm 10 \mathrm{~A}$ ), also functions as a slave to a second AMI Model 420 Power Supply Programmer unit. During magnet operations, the shim coils are energized oneby-one to their optimized current values. A LabView-based program installed on a PC functions as the interface between the user and the Model 420 Power Supply Programmer units.

The MWPC/plastic scintillator electron detector packages, described in detail below, are mounted to the two ends of the solenoidal magnet via $41.9-\mathrm{cm}$ conflat flanges. The central axis of the spectrometer is located $2.74 \mathrm{~m}$ above the experimental floor. 


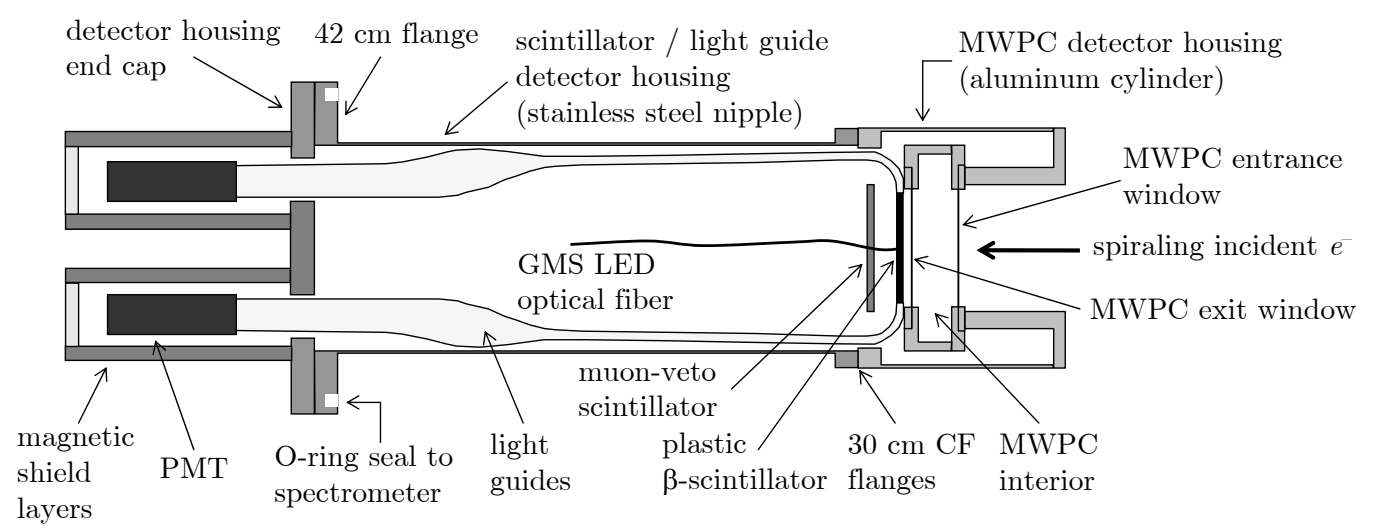

Fig. 3. Schematic diagram of the MWPC and plastic scintillator detector package.

\subsection{Multiwire proportional chambers}

A schematic diagram illustrating the main components of the MWPC/plastic scintillator detector package is shown in Fig. 3. As noted already, the MWPCs are relatively insensitive to gamma ray backgrounds, offer a low threshold for the detection of backscattering events, and permit reconstruction of the transverse $(x, y)$ coordinates of the $\beta$-decay events. Although described in detail in [12, for completeness we briefly review some of the MWPCs' most important features here.

First, to suppress missed backscattering events (again, those events depositing no energy above threshold in the MWPC), the entrance window separating the MWPC fill gas from the spectrometer vacuum was designed to be as thin as possible. Second, because of this thin entrance window requirement, the fill gas pressure was required to be as low as possible. The chosen fill gas, $\mathrm{C}_{5} \mathrm{H}_{12}$ (2,2-Dimethylpropane, or "neopentane"), a low- $Z$ heavy hydrocarbon, was shown to yield sufficient gain at a pressure of 100 Torr. The minimum window thickness for a 15-cm diameter window (equal to the diameter of the plastic scintillator, discussed below) shown to support a 100 Torr difference with a minimal leak rate (from pinholes) was $6 \mu \mathrm{m}$ of aluminized Mylar 3 reinforced by Kevlar fiber. Third, the MWPC mechanical size was constrained by the requirement that the MWPC housing fit within the solenoid's 35-cm warm bore diameter while also providing sensitivity to the entire $10-\mathrm{cm}$ diameter decay trap (projecting to a $10 / \sqrt{0.6}=12.9 \mathrm{~cm}$ diameter in the 0.6 Tesla field expansion region where the MWPC/scintillator is mounted). Finally, the anode plane was strung with $6410-\mu \mathrm{m}$ diameter gold-plated tungsten wires, and the cathode planes, separated from the anode plane by $10 \mathrm{~mm}$, were

3 Note that when the spectrometer is configured such that the ends of the decay trap are not closed off with thin foils, the nominal $6 \mu \mathrm{m}$ aluminized Mylar entrance window is replaced with a UCN-reflective ${ }^{58} \mathrm{Ni}$-coated aluminized Mylar window (to suppress contamination from neutron capture on ${ }^{27} \mathrm{Al}$ ). 
strung with $6450-\mu \mathrm{m}$ diameter gold-plated aluminum wires. The wire spacing on both the anode and the two cathode planes is $2.54 \mathrm{~mm}$, yielding an active area of $16.3 \times 16.3 \mathrm{~cm}^{2}$. This active area maps to a $12.6 \times 12.6 \mathrm{~cm}^{2}$ square in the 1.0-Tesla region, providing full coverage of the $10-\mathrm{cm}$ diameter decay trap.

All of the anode wires are connected via conductor tracks on the anode wireplane support frame; thus, the anode signal that is read-out is the summation of the signals on the 64 individual wires. With the wires on one cathode plane oriented "vertically", providing information on the "horizontal" coordinate (defined to be the $x$-coordinate), and the wires on the other cathode plane oriented "horizontally", providing information on the "vertical" coordinate ( $y$-coordinate), the $(x, y)$ position for each event is reconstructed from the center-of-gravity of the two cathode planes' signals.

Note that the 64 wires on each of the two cathode planes are read-out in groups of four. This four-wire-grouping reduced the number of electronics channels (thereby reducing the cost), but did not, as shown later, degrade the resulting position resolution, as the typical four-wire-group multiplicity is $\sim 3-4$ for the $368.1 \mathrm{keV}$ electrons from a ${ }^{113} \mathrm{Sn}$ conversion-electron source 4

\subsection{Plastic scintillator detectors}

\subsubsection{Scintillator}

The plastic scintillator detector (or, hereafter, the " $\beta$-scintillator") is a 15 cm diameter, 3.5-mm thick disk of Eljen Technology EJ-204 scintillator [23]. The EJ-204 wavelength of maximum emission is $408 \mathrm{~nm}$. This wavelength couples well to a typical bialkili photocathode's wavelength sensitivity, and is also sufficiently long for efficient optical transmission through a UVT light guide. Other desirable features of the EJ-204 scintillator which motivated its choice include a high light output ( $68 \%$ of anthracene), long attenuation length (1.6 m), fast rise time (0.7 ns), narrow pulse width (2.2 ns FWHM), and an index of refraction (1.58) well-matched to that of UVT light guide (1.49).

The 15-cm diameter in the 0.6-Tesla field-expansion detector region maps to a $15 \sqrt{0.6}=11.6 \mathrm{~cm}$ diameter disc in the 1.0-Tesla region of the spectrometer, providing full coverage of the 10-cm diameter decay trap. As discussed in [20], the 3.5-mm thickness was determined by two factors. First, the range of an $E_{0}=782 \mathrm{keV}$ endpoint energy electron in plastic scintillator is $3.1 \mathrm{~mm}$. Thus,

4 The $368.1 \mathrm{keV}$ energy we reference hereafter is the weighted mean (by intensity) of the ${ }^{113} \mathrm{Sn} \mathrm{K}-$, L-, and M-shell conversion-electron energies of $363.8 \mathrm{keV}$ (28.4\%), $387.5 \mathrm{keV}(5.6 \%)$, and $390.9 \mathrm{keV}(1.1 \%)$. The resolution of the scintillator is not sufficient to distinguish the individual lines. 
a total energy measurement requires a thickness of at least $3.1 \mathrm{~mm}$. Second, the probability of a gamma ray interaction in thin plastic scintillator is expected to scale with the scintillator volume. Thus, suppression of this background demands the thinnest possible scintillator 5 The chosen $3.5 \mathrm{~mm}$ thickness included an additional thickness "safety factor" for the energy measurement.

\subsubsection{Light guide system}

As indicated in Fig. 3, the light from the scintillator disc is transported out of the 0.6-Tesla field-expansion region over a distance of $\sim 1 \mathrm{~m}$ along a series of UVT light guides to photomultiplier tubes, situated in a region where the magnetic field is $\sim 300$ Gauss. Two options were considered for light collection from the scintillator: (a) coupling light guides directly to the scintillator back face, or (b) coupling light guides to the scintillator edge. Ultimately, edge collection was chosen for the following reasons. First, the surface area of the back face is a factor $\sim 10$ greater than the surface area of the edge, requiring an order-of-magnitude more photocathode coverage (leading to higher cost, and also higher dark noise), while only yielding $\sim 18 \%$ more light collection [20]. Second, cosmic-ray muons traversing the light guides generate Čerenkov light. Suppression of this background favored the smaller light guide volume associated with edge collection.

The design for the edge collection light guide system was constrained by the requirement that the detector system (shown in Fig. 31) fit within the spectrometer's 35-cm diameter warm bore, with an additional radial space allowance for cabling and efficient vacuum pumping. The design that was implemented, shown schematically in Fig. 4, consisted of twelve rectangular strips of 39-mm wide $\times 10$-mm thick UVT light guide coupled to the edge of the scintillator concentrically around its circumference (via optical grease). Each of these twelve strips includes a 5-mm straight section, which couples the 3.5-mm thick scintillator edge to the $10-\mathrm{mm}$ thick light guide. Following the 5 -mm straight sections, the light guides are then bent through $90^{\circ}$ over a $35-\mathrm{mm}$ bend radius and directed towards the photomultiplier tubes. A novel feature of the light guide transport system is that the twelve rectangular strips adiabatically transform into four $39 \times 30 \mathrm{~mm}^{2}$ rectangular clusters, which are then coupled to the (four) photomultiplier tubes. The entire light guide system is supported by an aluminum ring-like support frame. A photograph of the as-built light guide system, manufactured by Suzuno Giken [24], appears in Fig. 5.

\footnotetext{
5 For the 3.5-mm thickness, the typical energy deposition from cosmic-ray muons in the $\beta$-scintillator is $\sim 700-1500 \mathrm{keV}$ (i.e., the peak is very broad). Increasing the scintillator thickness would have shifted the muon "peak" past the neutron $\beta$ decay endpoint, but would have increased the total background rate from gamma rays. Note that muons are vetoed with a scintillator located immediately behind the $\beta$-scintillator (see Fig. 33).
} 


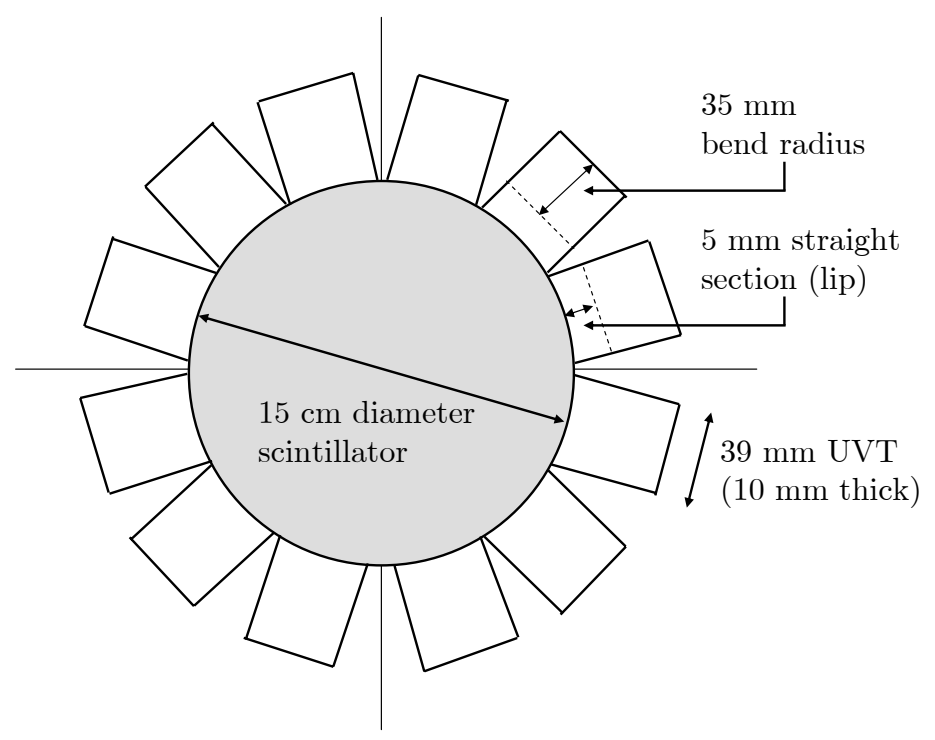

Fig. 4. Schematic diagram (front view) of the light guide system at the scintillator edge interface.

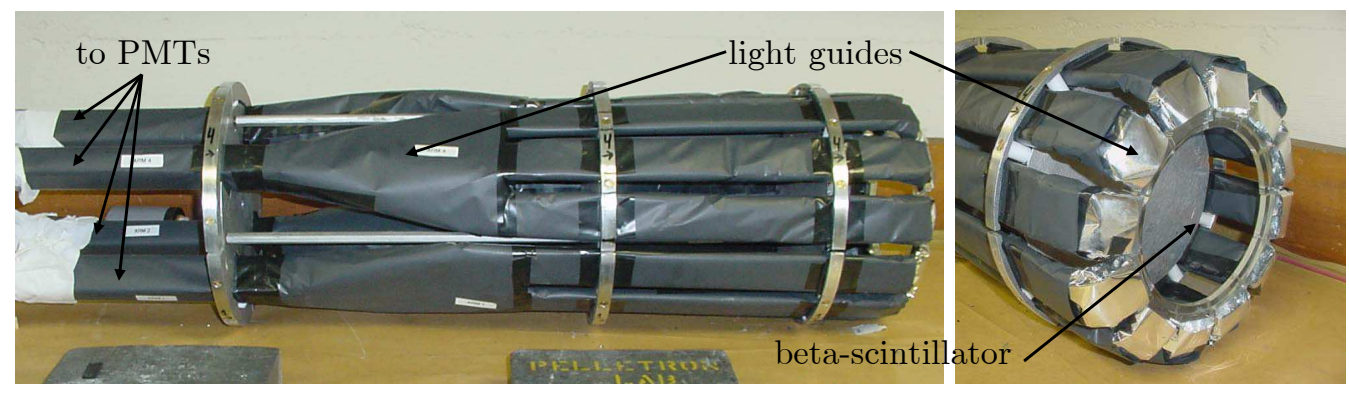

Fig. 5. (Color online) Photograph of the $\beta$-scintillator and light guide assembly.

\subsubsection{Photomultiplier tubes}

The choice of readout by four photomultiplier tubes (PMTs) was motivated by the following considerations. The effective light collection surface area of the twelve rectangular light guides at the scintillator edge interface is $12 \times(39 \times$ $\left.10 \mathrm{~mm}^{2}\right)=4680 \mathrm{~mm}^{2}$. This surface area maps onto thirteen 1-inch PMTs, three 2-inch PMTs, or one 3-inch PMT. Readout by more than one PMT was desired, as the requirement of a multi-PMT coincidence suppresses dark noise and backgrounds from gamma-ray interactions in the scintillator and Cerenkov light from cosmic-ray muons traversing a set of light guides coupled to the same PMT. Ultimately, readout with four 2-inch PMT 6 was chosen as it provides for a multi-PMT coincidence, but requires fewer electronics channels than readout with 1-inch PMTs.

$\overline{6}$ Note that four (as opposed to three) 2-inch PMTs are actually required in order to couple the rectangular geometry of the light guides to the circular geometry of the photocathodes. 


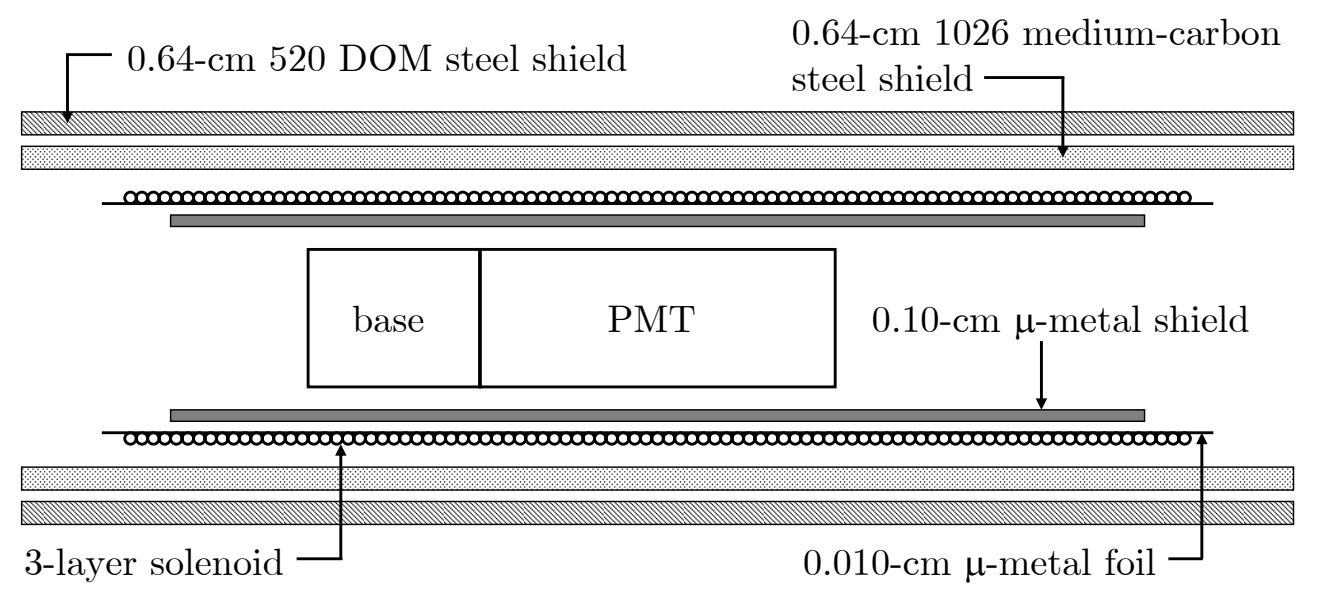

Fig. 6. Schematic diagram of the magnetic shielding for the photomultiplier tubes. See text for explanation of layers.

The Burle 8850, a 12-stage photomultiplier with a bialkili photocathode and a high-gain gallium-phosphide first dynode [25], was chosen for the experiment. This PMT was favored as it is optimized for single-photon counting and features a low dark noise rate, a fast rise time of $2.1 \mathrm{~ns}$, a high gain of $1.6 \times 10^{7}$ at a nominal operating voltage of $2000 \mathrm{~V}$ (well below its maximum operating voltage of $3000 \mathrm{~V}$ ), and a peak quantum efficiency at $420 \mathrm{~nm}$ (well matched to the EJ-204 scintillator's $408 \mathrm{~nm}$ wavelength of maximum emission). The PMTs are glued to the ends of the rectangular light guides. High voltage for the PMTs is supplied with a LeCroy HV4032 power supply unit.

\subsubsection{Photomultiplier tube magnetic shielding}

The magnetic field in the region of the PMTs is oriented primarily in the axial direction, with a magnitude of $\sim 300$ Gauss. This field is shielded with an array of active and passive magnetic shielding components, shown schematically in Fig. 6. Moving from the exterior to the interior, the first element is a 0.64cm-thick 520 DOM steel shield, with a nominal outer diameter of 11.4-cm. The next element is a close-fitting 0.64-cm-thick 1026 medium-carbon steel shield with a nominal outer diameter of $10.2-\mathrm{cm}$. The lengths of both of these steel shields are $55.9-\mathrm{cm}$. The third and fourth layers consist of a $\sim 37.6-\mathrm{cm}-$ long 3-layer $\sim 325$-turn solenoid wound (with 18 AWG copper magnet wire) on the surface of a 7.6-cm outer diameter, 0.10-cm-thick cylinder of Advance Magnetics AD-MU-80 $\mu$-metal. Note that because the $\mu$-metal shield is only 30.5-cm in length, a 38.1-cm-long, 0.010-cm-thick sheet of AD-MU-80 $\mu$-metal foil was wrapped on the surface of the 30.5 -cm-long $\mu$-metal cylinder, with the solenoids then actually wound on the surface of the thin $\mu$-metal foil.

Bench tests of this magnetic shielding configuration with fields comparable to the spectrometer fields in the region of the PMTs (i.e., up to $\sim 200-400$ Gauss) showed that residual shielded fields of $\sim 0.1$ Gauss could be achieved 
with solenoid currents no greater than 1.0 A. Magnetic end caps were not required.

\subsection{Detector housing and spectrometer mount}

The combined MWPC/plastic scintillator detector package is mounted within a detector housing and inserted into the warm bore of the 1-Tesla solenoidal spectrometer. After insertion, the $\beta$-scintillator is located $2.2 \mathrm{~m}$ from the center of the UCN decay trap. As shown on the detector schematic in Fig. 3, the $\beta$ scintillator and the light guide assembly are enclosed within a $\sim 64$-cm-long, $26.7-\mathrm{cm}$ outer diameter stainless steel nipple with a $30.5-\mathrm{cm}$ conflat flange welded to one end, and a 41.9-cm flange (with an O-ring groove) welded to the other end. The MWPC is mounted to the nipple via $30.5-\mathrm{cm}$ conflat flanges. The 41.9-cm flange on the other end of the nipple is mounted to the $41.9-\mathrm{cm}$ conflat flange on the spectrometer warm bore.

Also shown on the schematic in Fig. 3 are the magnetic shields for the PMTs, which extend beyond the 41.9-cm flange on the end of the nipple. The next-tooutermost shield layers, the 0.64-cm-thick 1026 medium-carbon steel shields, also serve as the vacuum seal (via O-ring grooves) for the region beyond the 41.9-cm flange on the nipple.

\subsection{Gas flow and vacuum system}

A schematic of the gas flow and vacuum system for the MWPC and $\beta$ scintillator detector assembly is shown in Fig. 7. The MWPC volume (nominally, 100 Torr of neopentane) is separated from the spectrometer vacuum by the MWPC entrance window. The detector housing for the $\beta$-scintillator, light guide, and PMT assembly (nominally, $\sim 95$ Torr of nitrogen 7 ) is separated from the MWPC volume by the MWPC exit window. During normal operation, the spectrometer, MWPC, and scintillator detector housing are first evacuated at a rate of $<1$ Torr/s, to minimize pressure gradients across the MWPC windows. Pressures in the spectrometer volume of $\sim 10^{-6}$ Torr are typical. The MWPC and scintillator detector housing are then filled (at a rate significantly less than 1 Torr/s) with neopentane and nitrogen, respectively, to their nominal operating presures with the gas handling system described

$\overline{7}$ As noted in [12, the pressure of the nitrogen in the scintillator detector housing is maintained at a somewhat lower pressure than the MWPC pressure. This is to ensure that the MWPC exit window bows out, or away, from the MWPC interior, avoiding contact with the MWPC wire planes. 


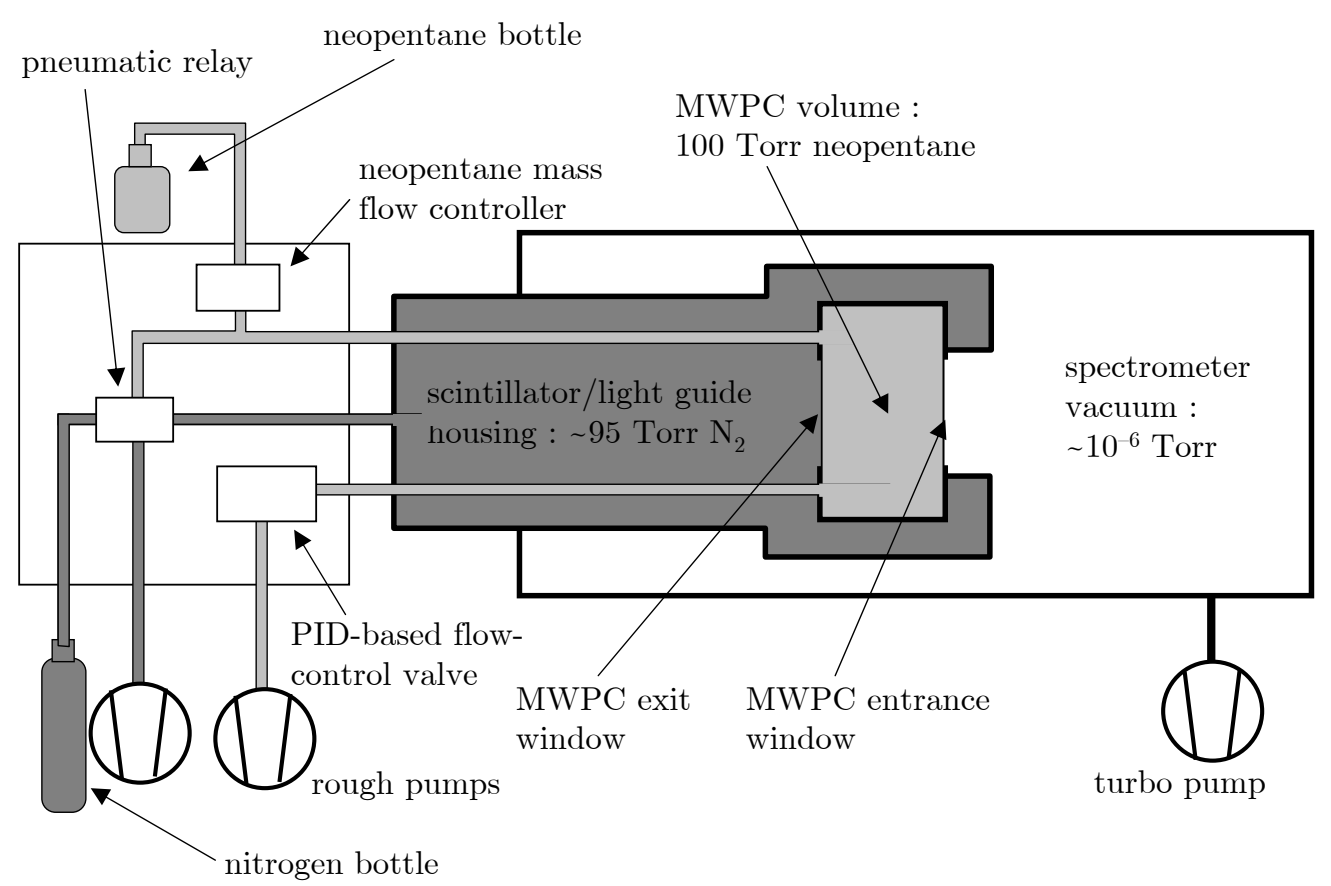

Fig. 7. Schematic diagram of the gas flow and vacuum system for the MWPC and $\beta$-scintillator detector package coupled to the spectrometer vacuum.

in [12]. Also shown in [12] is a photograph of the entire MWPC/ $\beta$-scintillator assembly on a rolling cart.

\subsection{Gain monitoring system}

Possible temporal shifts in the $\beta$-scintillator's photomultiplier tubes' gains are monitored, and subsequently corrected for, with a gain monitoring system (GMS). Separate (but identical) GMS systems were constructed for the two $\beta$-scintillators. Note that these two separate systems are not redundant, as the gains of the PMTs in the two detector packages can vary independently. An overview of the GMS system is as follows.

A Nichia Corporation UV LED (Model NSHU590) is enclosed within a lighttight aluminum box. The LED is driven in pulsed mode by a voltage pulse from a CAEN Model C 529 (6-channel) LED driver installed in a CAMAC crate, and controlled by a PC laptop. The LED light is transported along two fiber optic cables to the $\beta$-scintillator (as indicated in Fig. 31) and to a "monitor" setup consisting of a NaI crystal viewed by a PMT. A gamma ray source $\left({ }^{60} \mathrm{Co}, 1.173 \mathrm{MeV}\right.$ and $1.333 \mathrm{MeV}$ lines) is located nearby. The ${ }^{60} \mathrm{Co}$ spectrum measured by the monitor PMT provides a reference point for the stability of the amplitude of the LED output pulse. Variations in the LED spectra recorded in the $\beta$-scintillator PMTs are then referenced to variations in the LED spectrum relative to the ${ }^{60} \mathrm{Co}$ spectrum observed in the monitor 
PMT.

It can then be shown that the gain correction for some $\beta$-scintillator PMT, $G_{\beta}(t)$, at some particular time $t$ relative to some starting time $t=0$ is

$$
G_{\beta}(t)=\left(\frac{\operatorname{LED}_{m}(t)}{\operatorname{LED}_{m}(0)}\right)\left(\frac{{ }^{60} \mathrm{Co}_{m}(0)}{{ }^{60} \mathrm{Co}_{m}(t)}\right)\left(\frac{\operatorname{LED}_{\beta}(0)}{\operatorname{LED}_{\beta}(t)}\right)
$$

Here, $\operatorname{LED}_{m}(\ldots)$ denotes the response of the monitor PMT to the LED pulse, ${ }^{60} \mathrm{Co}_{m}(\ldots)$ the response of the monitor PMT to the ${ }^{60} \mathrm{Co}$ gamma ray spectrum, and $\operatorname{LED}_{\beta}(\ldots)$ the response of the $\beta$-scintillator PMT to the LED pulse. The referencing of the LED response to the ${ }^{60} \mathrm{Co}$ response compensates for fluctuations in the LED output. Note that drifts in the gain of the monitor PMT are also compensated, as the ${ }^{60} \mathrm{Co}$ and LED spectra shift proportionally.

\subsection{Electronics and data acquisition}

\subsubsection{Event trigger logic}

The electronics for the experiment consists of a VME system for the event trigger logic (via discriminators and programmable logic units) and the readout of scalers, analog-to-digital convertors (ADCs), and time-to-digital convertors (TDCs). A NIM system coupled to the VME system is employed for the implementation of a "busy logic". This busy logic vetos event triggers arriving during ADC and TDC conversion times (i.e., while the ADC and TDC states are "busy"), and was implemented to prevent re-triggering (and subsequent readout of the ADCs and TDCs) by scintillator afterpulses (single photoelectron pulses from the slow-time component of the scintillator light emission). Bench studies showed that a veto time of $\sim 1 \mu$ s was sufficient to completely suppress contamination from re-triggering by afterpulses [20].

A schematic diagram of the trigger logic is shown in Fig. 8, and an overview is as follows. For each detector package (detector 1 or detector 2), a trigger is defined by a two-fold PMT coincidence requiring at least two PMTs with analog signals above the discriminator threshold. Note that the discriminator threshold for each PMT is set at $\sim 1 / 2$ of the amplitude of its single photoelectron peak. An OR of a detector 1 two-fold coincidence trigger, detector 2 two-fold coincidence trigger, global GMS LED trigger, detector 1 GMS monitor trigger (from ${ }^{60} \mathrm{Co}$ events), detector 2 GMS monitor trigger $\left({ }^{60} \mathrm{Co}\right)$, and other experiment triggers then generates the main event trigger. The trigger logic for the two detectors' two-fold coincidences and the main event trigger are performed with CAEN V495 Dual Programmable Logic Units (PLUs). Those event triggers that are not vetoed by the busy logic then trigger gate/delay generators 


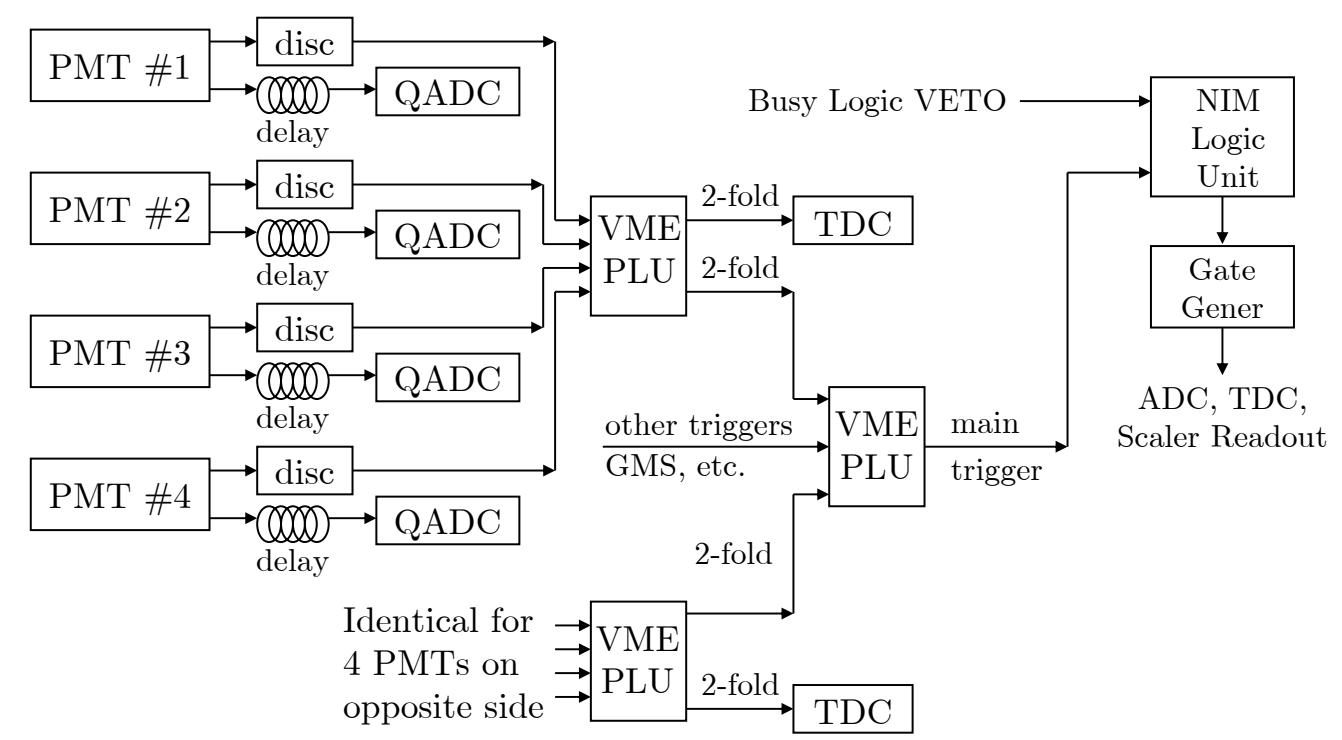

Fig. 8. Simplified schematic diagram of the trigger logic. A significant number of modules and circuit paths have been omitted. As noted in the text, the "main trigger" is an OR of all possible event triggers.

for the readout of the ADCs, TDCs, and scaler modules.

Among other variables, the TDCs (CAEN V775 modules with a 32-event buffer memory, operated in COMMON STOP mode) provide a relative measurement of the two detectors' two-fold coincidence trigger arrival times. Such relative timing information permits identification of the earlier trigger (if two such triggers arrive), important for the correct assignment of the initial direction of incidence for backscattering events triggering both $\beta$-scintillators. Two types of ADC modules are used. A charge-integrating ADC (CAEN V792 QADC module with a 32-event buffer memory), triggered for readout by a $\sim 140 \mathrm{~ns}$ gate from a CAEN V486 gate/delay generator, provides a measurement of the total charge measured in each PMT. The sum of the QADC spectra from the four PMTs on each side is proportional to the energy deposition in the scintillator. Peak-sensing ADCs (CAEN V785 PADC modules with a 32-event buffer memory), triggered for readout by a $\sim 6 \mu$ s gate from a CAEN V462 gate generator, digitize the MWPC anode and cathode-plane signals. Scalers (CAEN V820 and V830 modules; the V830 module features a 32-event buffer memory) are used for deadtime monitoring.

System deadtimes were measured offline as a function of the trigger rate with a random pulser and found to be smaller than $10 \%$ for event rates up to $\sim 5 \mathrm{kHz} 8$ At lower rates of $\sim 0.5-1.0 \mathrm{kHz}$, the deadtime is proportional to the event rate, of order $\sim 1 \%$, and dominated by the ADC and TDC digitization times.

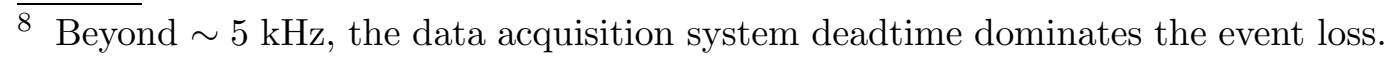




\subsubsection{Data acquisition}

The data acquisition system is based on the MIDAS package [26]. Among many components, MIDAS features a buffer manager for data flow management, a frontend acquisition code, a data logger for the storage of data on disk, online analysis capabilities, a web-browser-based run control, and an alarm system. The UCNA experiment utilizes a dedicated Linux-based workstation for implementation of the frontend acquisition code, and another separate Linux-based workstation for run control and online analysis. The frontend acquisition code accesses the VME system via a Struck PCI/VME interface 9 The frontend continually polls the data-ready status of the ADCs and TDCs. Whenever any of the ADCs and/or TDCs have data ready for readout in memory, the raw data from all ADC, TDC, and scaler modules are transferred to the data buffer manager. The MIDAS event format structure consists of "banks" of raw data, which are subsequently decoded by the MIDAS analyzer into formats readable by the CERNLIB analysis packages ROOT and PAW (i.e., "Ntuples").

\section{Results from spectrometer performance studies}

In this section we show select results from performance studies of the electron spectrometer carried out with a source of ${ }^{113} \mathrm{Sn} 368.1 \mathrm{keV}$ conversion electrons with an event rate (above background) as measured in the spectrometer of $\sim 85 \mathrm{~Hz}$. The principal analysis results shown below include energy spectra, position spectra, an assessment of the spectrometer's position resolution, and an assessment of the spectrometer's instrumental asymmetry.

\subsection{Solenoidal field map}

First, however, we show results from field maps of the 1.0-Tesla region (measured with an NMR probe) in Fig. 9. As can be seen there, the field profile satisfies the $\pm 5 \times 10^{-4}$ field uniformity requirement over the 3-m extent of the UCN decay trap region. Figure 10 displays a field map of the 1.0-Tesla to 0.6-Tesla field-exansion region (measured with a Hall probe).

9 A Struck SIS1100 PCI card hosted by the Linux workstation running the frontend acquisition code is connected via a fiber optic link to a Struck SIS3100 VME board hosted by the VME crate. 


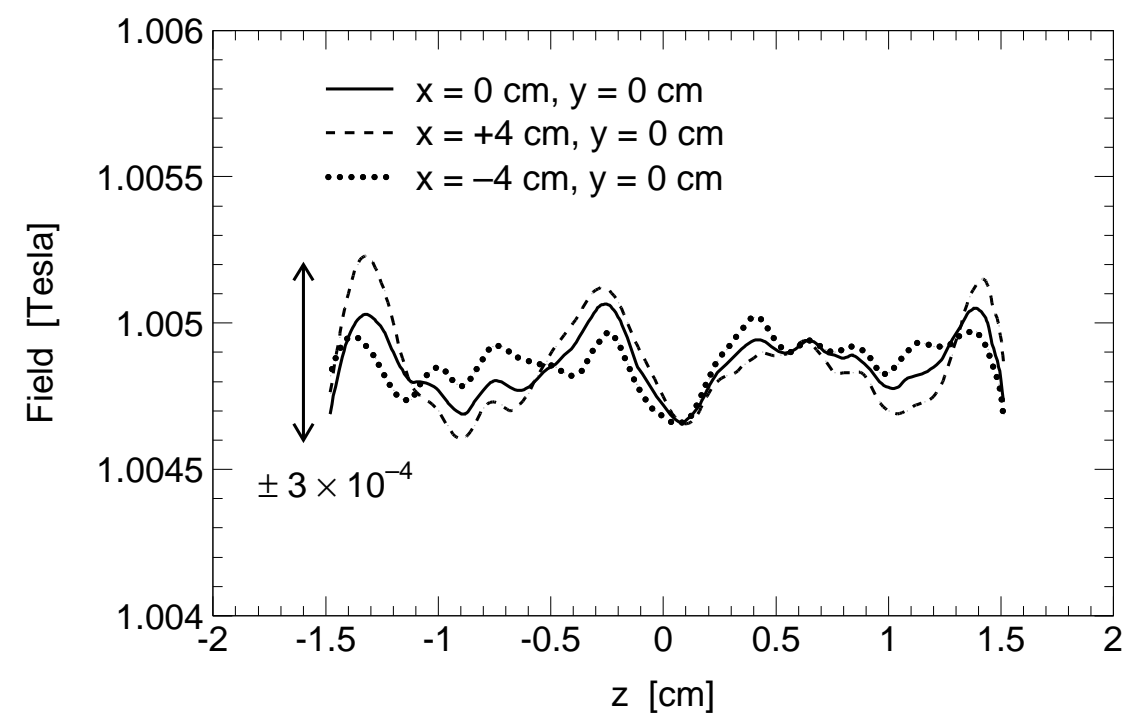

Fig. 9. Field maps from three different scans along the UCN decay trap's 3-m length, showing uniformities at the level of $\pm 3 \times 10^{-4}$.

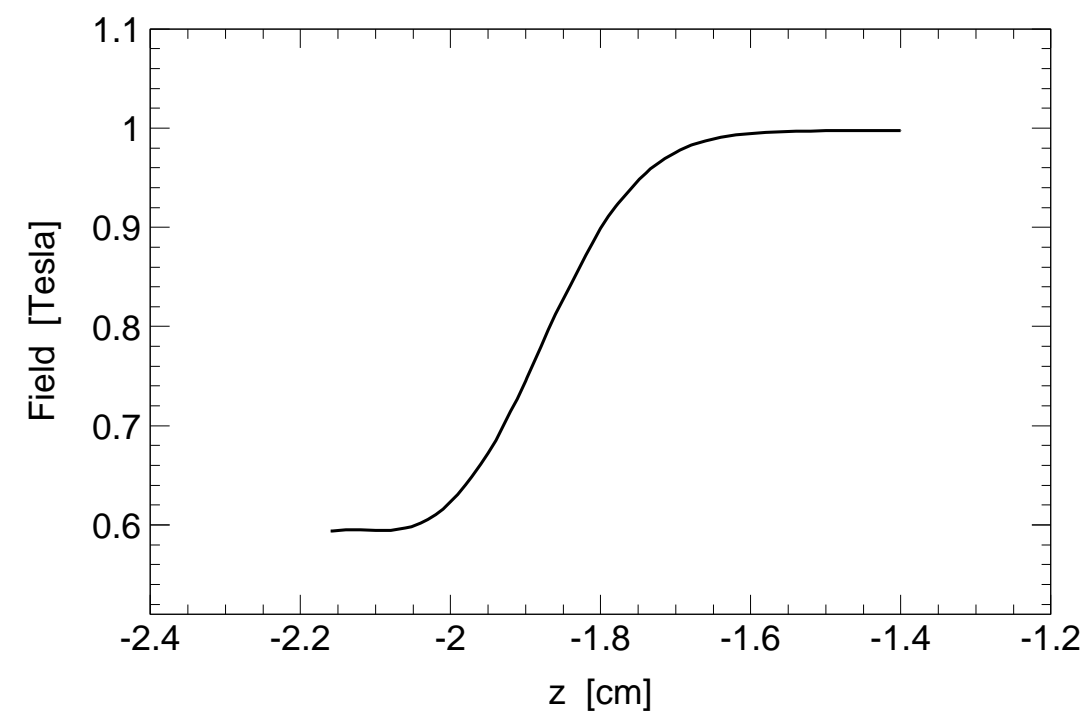

Fig. 10. Field map of the 1.0-Tesla to 0.6-Tesla field-expansion region.

\subsection{Energy spectra}

A typical MWPC anode spectrum extracted from a run taken with the ${ }^{113} \mathrm{Sn}$ source positioned with the spectrometer's fiducial volume is shown in Fig. 11 . The $\beta$-scintillator spectrum (summed over the response of the four photomultiplier tubes) for this same run, shown before and after application of the cut on the MWPC anode response, is shown in Fig. 12. As can be seen there, the requirement of a coincidence between the MWPC and the $\beta$-scintillator greatly reduces the background from gamma-ray interactions. The low-energy tail on the ${ }^{113} \mathrm{Sn}$ spectrum is primarily the result of energy loss in the MWPC 


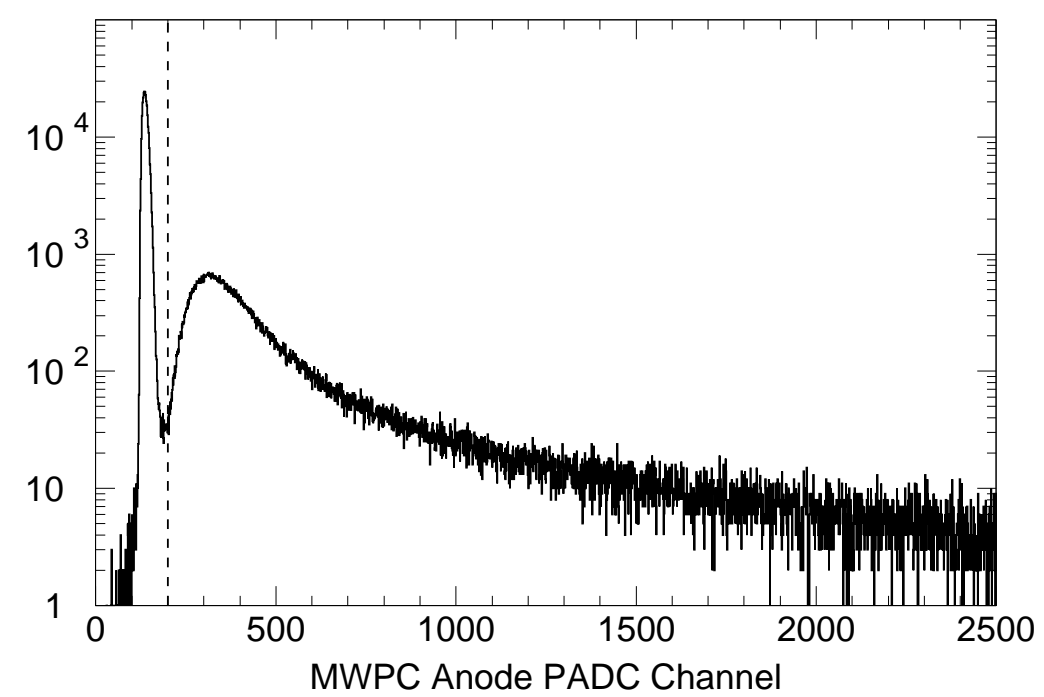

Fig. 11. Typical MWPC anode spectrum (PADC channel number) from a run with the ${ }^{113} \mathrm{Sn}$ source positioned within the fiducial volume. The dashed line indicates the typical position of the cut.

entrance and exit windows 10 Backscattering from the MWPC/ $\beta$-scintillator also contributes to the tail, but the contribution of this process to the structure of the tail is small (as the total detected backscattering event fraction is small, $\sim 4.6 \%$ for the $368.1 \mathrm{keV}{ }^{113} \mathrm{Sn}$ conversion electron energies) compared to the energy loss in the MWPC windows.

The impact of energy loss in the MWPC windows is illustrated in Fig. 13. There, results from a GEANT4 simulation of the energy loss in the MWPC windows are plotted versus the simulated visible energy 11 deposition in the $\beta$-scintillator, and overlaid with a histogram of the visible energy deposition. Although not shown there, the energy loss in the MWPC windows is correlated with the initial pitch angle in the 1.0-Tesla region.

\subsection{Event types}

\subsubsection{Definitions}

The $\beta$-asymmetry measurement requires an accurate determination of the electron's initial direction of incidence. Complicating the extraction of the asymmetry are the five different event types, shown schematically in Fig. 14.

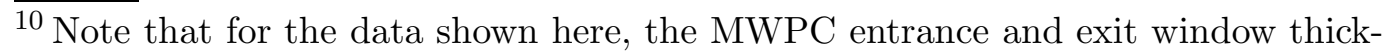
nesses were $25 \mu \mathrm{m}$ Mylar (non-aluminized), not the optimized $6 \mu \mathrm{m}$ thickness.

${ }^{11}$ The visible energy is defined to be the total energy loss in the $\beta$-scintillator active region (beyond the dead layer) for those events generating a two-fold PMT coincidence trigger.
} 


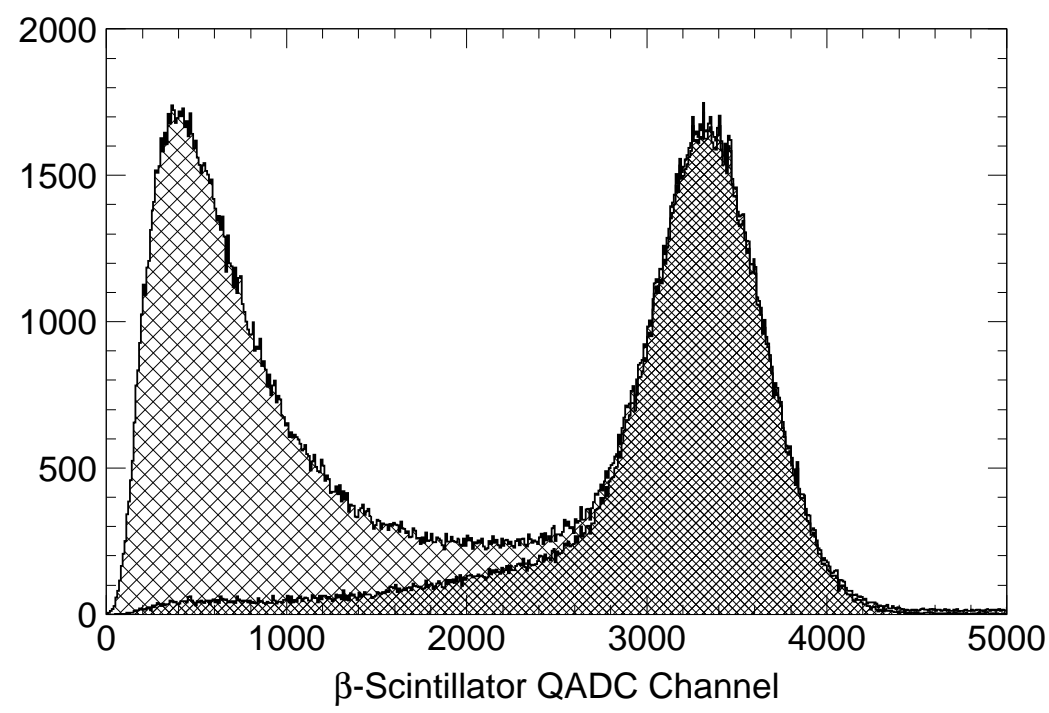

Fig. 12. Typical $\beta$-scintillator spectra from a run with the ${ }^{113} \mathrm{Sn}$ source positioned within the fiducial volume. The light-shaded (dark-shaded) histogram shows the spectrum before (after) the cut on the MWPC anode response.

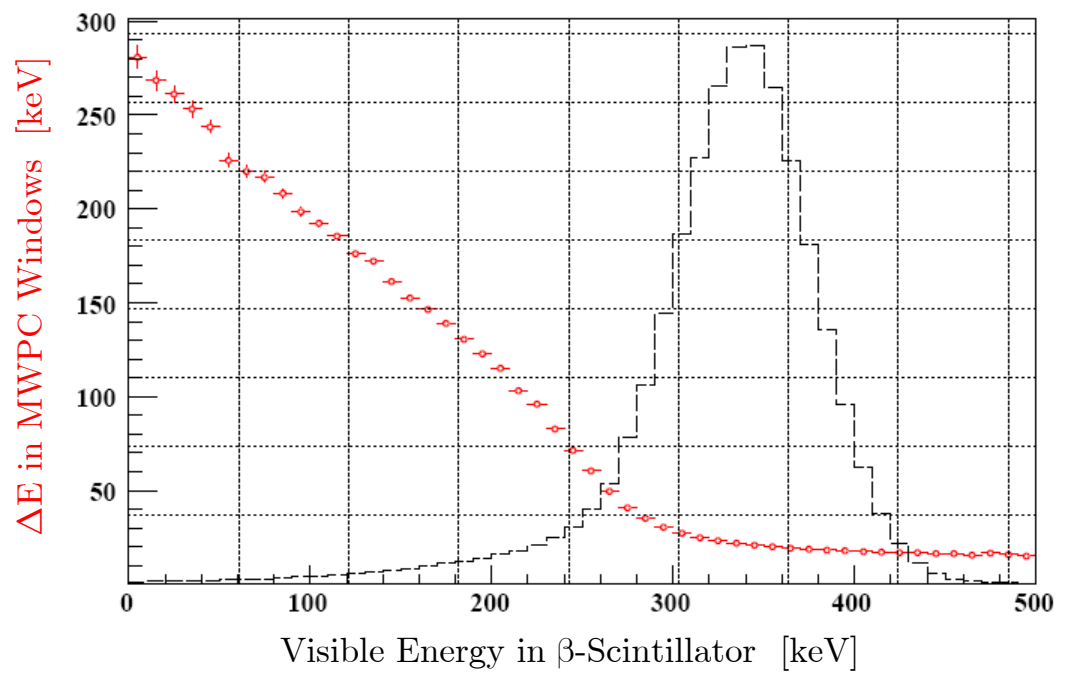

Fig. 13. GEANT4 simulated results for energy loss from ${ }^{113} \mathrm{Sn}$ conversion electrons in the MWPC windows plotted versus the visible energy deposition in the $\beta$-scintillator, overlaid with a histogram of the visible energy distribution.

- Correct events: Events in which an electron, incident initially on one of the $\beta$-detector packages, does not backscatter from any element of that side's $\beta$-detector package, and is then detected in that side's $\beta$-scintillator.

- Type I backscattering events: Events in which an electron, incident initially on one of the $\beta$-detector packages, deposits sufficient energy to generate a two-fold trigger in that side's $\beta$-scintillator, backscatters from that side's $\beta$-scintillator, and is then detected in the opposite side's $\beta$-scintillator.

- Type II backscattering events: Events in which an electron, incident intially on one of the $\beta$-detector packages, deposits energy above the cut in that 


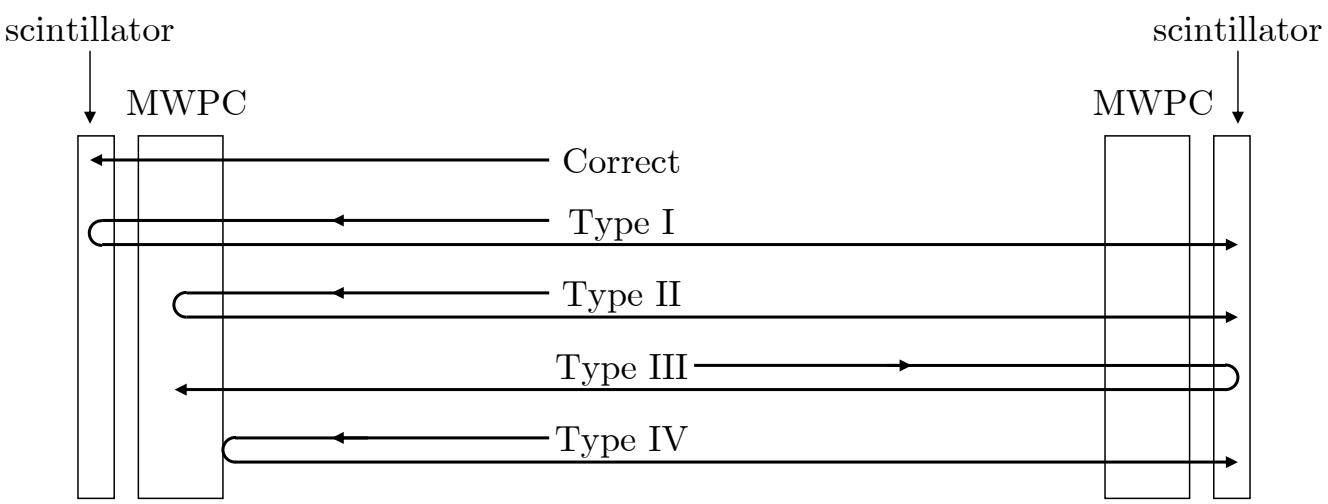

Fig. 14. Schematic diagram of the five event types. See text for descriptions.

side's MWPC, backscatters either from that side's MWPC (i.e., gas, wire planes, or back window) or $\beta$-scintillator without depositing sufficient energy to generate a two-fold trigger (e.g., backscatters from the scintillator's dead layer), and is then detected in the opposite side's $\beta$-scintillator.

- Type III backscattering events: Events in which an electron, incident initially on one of the $\beta$-detector packages, deposits sufficient energy to generate a two-fold trigger in that side's $\beta$-scintillator, backscatters from that side's $\beta$-scintillator, deposits energy above the cut in the opposite side's MWPC, and is then stopped in either the opposite-side MWPC's gas, wire planes, or back window or the opposite-side's $\beta$-scintillator's dead layer.

- Type IV backscattering events: Events in which an electron, incident initially on one of the $\beta$-detector packages, backscatters from that side's MWPC without depositing energy above the cut (e.g., backscatters from the MWPC entrance window), and is then detected in the opposite side's $\beta$-scintillator.

Note that Type I backscattering events can be identified via examination of the two detectors' two-fold coincidence trigger arrival times, thus determining the initial direction of incidence unambiguously (as discussed earlier in Section 3.7.1). In contrast, simulation input (regarding the energy deposition in the MWPCs) is needed to distinguish Type II from Type III backscattering events. For example, without using any such simulation input (i.e., using only the $\beta$ scintillator trigger to determine the direction of incidence), the Type II and Type III events shown in Fig. 14 would appear identical, with the direction of incidence for the Type II event misidentified. Type IV events cannot, of course, be distinguished from Correct events; these are the "missed backscattering events" discussed earlier in Section 2.4 which can only be corrected for in simulation.

The analysis results presented hereafter are only for those events identified by the event reconstruction as Correct events. Because this event sample also includes (unidentifiable) Type IV backscattering events, we label these events "Type 0 events". As noted earlier, a detailed companion paper discussing the identification of, and energy spectra of, the various backscattering event types 


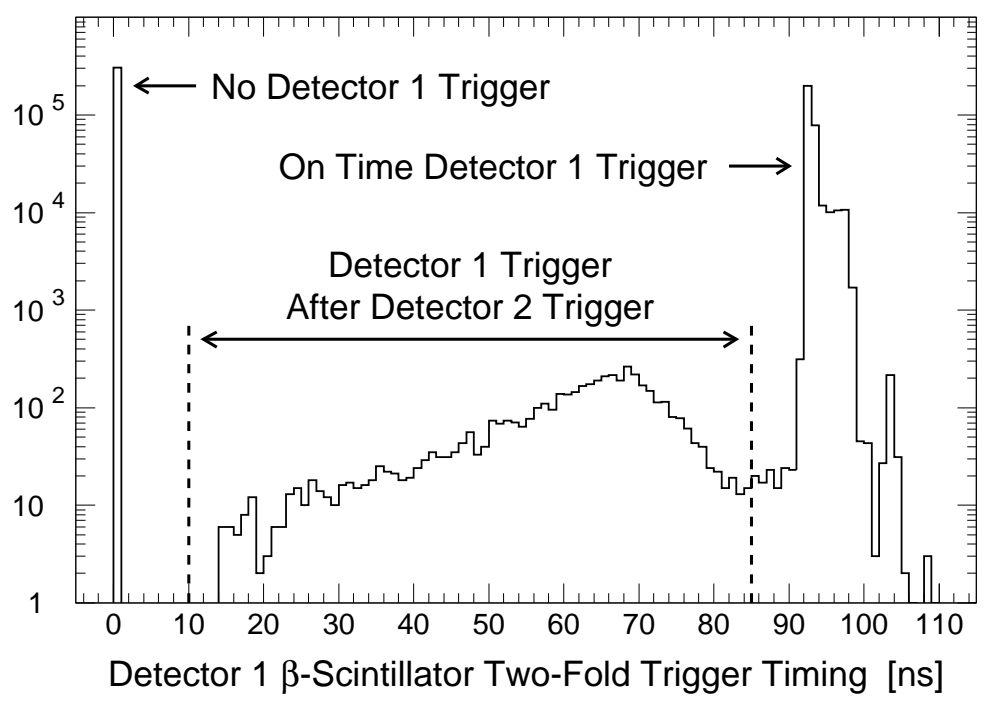

Fig. 15. Typical two-fold coincidence trigger arrival timing spectrum. See text for interpretation of the peaks.

is in preparation [19].

\subsubsection{Selection of Type 0 events}

The trigger logic (shown in Fig. 8) is such that each two-fold trigger generates the START for its respective TDC channel. The main event trigger (as noted earlier, an OR of the two-fold triggers and any other event triggers) is delayed by $\sim 92$ ns and generates the COMMON STOP for the TDC. A typical two-fold coincidence arrival time spectrum (shown here for detector 1 two-fold triggers) at the main trigger logic unit is shown in Fig. 15. Main event triggers generated by the arrival of a detector 1 two-fold trigger appear at the delay time of $\sim 92$ ns (i.e., an "on time" trigger), whereas main event triggers generated by the arrival of a detector 2 two-fold trigger, with no later arrival of a detector 1 two-fold trigger, appear at the peak at 0 ns (i.e., a "time-out" for that TDC channel). Those events located between these two extremes (i.e., the broad secondary peak located at $\sim 68 \mathrm{~ns}$ ) correspond to main event triggers generated by the arrival of a detector 2 two-fold trigger at the logic unit, with the later arrival of a detector 1 two-fold trigger. In other words, these are events which backscattered from the detector 2 package into the detector 1 backage (i.e., Type I backscattering events incident initially on the detector 2 package). Note that a $150 \mathrm{keV}$ electron will traverse the $4.4 \mathrm{~m}$ separation between the two $\beta$-scintillators in $23.2 \mathrm{~ns}$; thus, the $\sim 24 \mathrm{~ns}$ separation between these two peaks is as expected. Note that the spectrum is cut off below $\sim 15$ ns because the dynamic range of the TDC was set at its minimum value of $140 \mathrm{~ns}$ for this run.

Type 0 events were identified according to the following selection rules: 


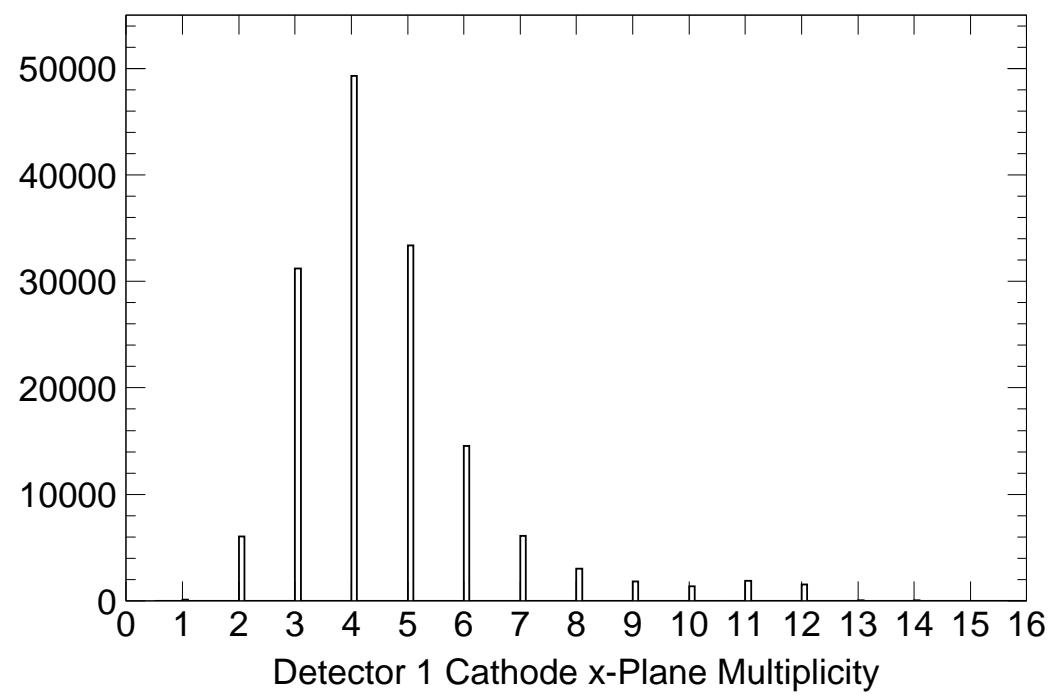

Fig. 16. Typical cathode-plane multiplicity (shown here for the detector $1 x$-plane) from a run with the ${ }^{113} \mathrm{Sn}$ source.

- The initial incidence detector package's two-fold coincidence trigger arrival time must reconstruct to the $\sim 92$ ns delay time. The other detector package's two-fold coincidence trigger arrival time must reconstruct to a TDC "time-out".

- The MWPC on the $\beta$-scintillator hit side must display an anode signal above the cut (as indicated in Fig. 11). The MWPC on the other side must display an anode signal below the cut.

- The muon-veto scintillator (as shown in Fig. 3) on the $\beta$-scintillator hit side must not record a hit.

\subsection{Position spectra}

\subsubsection{Cathode-plane multiplicities}

As noted earlier, the 64 wires (with 2.54-mm spacing) on each of the two MWPC cathode planes are read-out in groups of four. Henceforth, we will refer to each of these four-wire configurations simply as a "wire". Despite this four-wire grouping, most of the events exhibit a multiplicity (on each cathode plane) of $\geq 3$, where we define the event-by-event cathode multiplicity to be the number of wires with a signal above a prescribed threshold. A typical distribution of multiplicities from a run with the ${ }^{113} \mathrm{Sn}$ source is shown in Fig. 16. Here, the threshold was defined by a two-sigma limit ( $\sim 50$ channels $)$ of the cathode pedestal peaks, as determined by Gaussian peak fitting. 

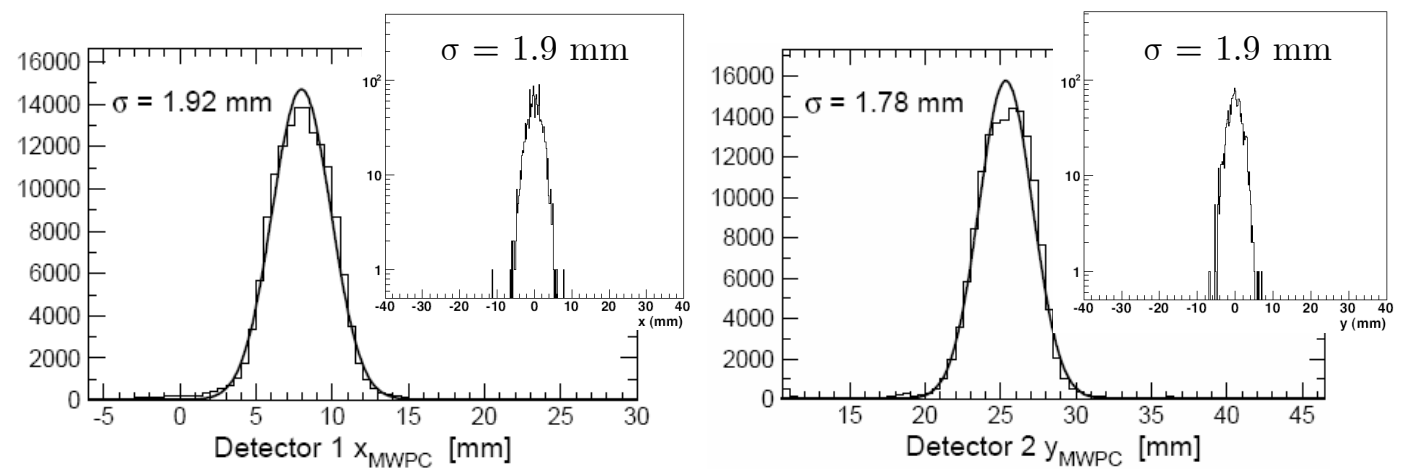

Fig. 17. Typical MWPC reconstructed position spectra for Type 0 events from a run with the ${ }^{113} \mathrm{Sn}$ source located within the fiducial volume. The solid curves are the results of Gaussian fits to the peaks, with the fitted widths indicated. Distributions obtained from a GEANT4 simulation of the MWPC response are shown in the insets.

\subsubsection{Position reconstruction results}

The transverse $(x, y)$ positions are computed from the cathode-plane wire spectra according to weighted sums,

$$
x_{\mathrm{MWPC}}=\frac{\sum_{i} Q_{i} x_{i}}{\sum_{i} Q_{i}}, \quad y_{\mathrm{MWPC}}=\frac{\sum_{j} Q_{j} y_{j}}{\sum_{j} Q_{j}},
$$

where $Q_{i}$ denotes the signal above pedestal for the $i^{\text {th }}$ channel, $x_{i}$ and $y_{i}$ denote the $x$ - and $y$-coordinate of the $i^{\text {th }}$ channel, and the sums $i$ and $j$ run over all of the $x$ - and $y$-plane cathode channels, respectively, satisfying the two-sigma pedestal threshold.

Sample MWPC reconstructed position spectra (shown here for detector $1 x$ reconstruction and detector $2 y$-reconstruction) for Type 0 events, extracted from a run with the ${ }^{113} \mathrm{Sn}$ source located within the fiducial volume, are shown in Fig. 17. Note that the position spectra shown here, and all spectra shown hereafter, have been projected back to the spectrometer's 1.0-Tesla region. Gaussian fits to the peaks are plotted, showing fitted widths slightly better than $\sim 2 \mathrm{~mm}$. The widths of these distributions agree well with those extracted from a GEANT4 Monte Carlo simulation of the MWPC response to the $368.1 \mathrm{keV}{ }^{113} \mathrm{Sn}$ conversion electrons (shown in the insets).

A two-dimensional plot of detector $1(x, y)$ positions are shown in Fig. 18 for those events identified by the muon-veto scintillator as muons. As expected, these background events are distributed uniformly over the MWPC's sensitive area. 


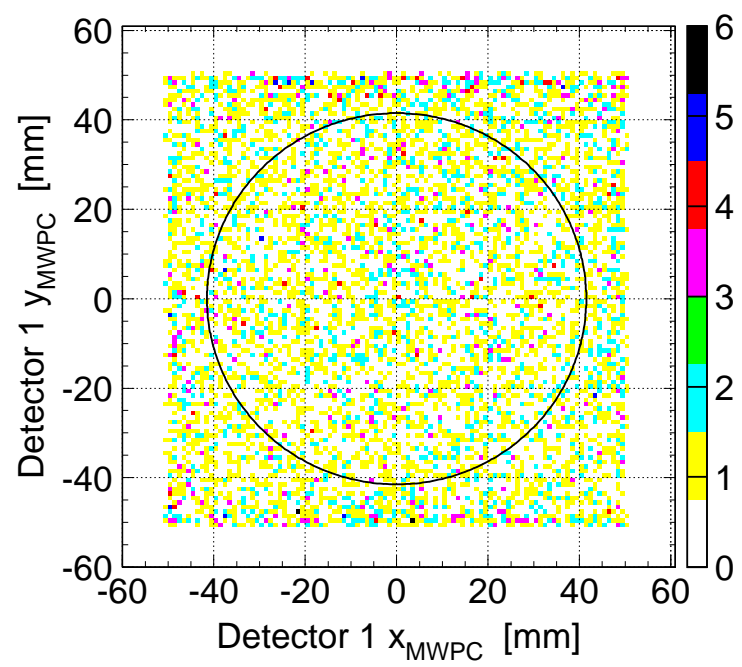

Fig. 18. (Color online) Two-dimensional plot of detector $1(x, y)$ positions for events tagged as muons by the muon-veto scintillator. The circle denotes the extent of the 41.5-mm radius fiducial volume.

\subsection{Spectrometer position resolution}

To assess the position response of the spectrometer, we analyzed data from runs taken with the ${ }^{113} \mathrm{Sn}$ source located at 96 different positions within the fiducial volume. We extracted the position resolution of the spectrometer from distributions of differences between the nominal (known) positions, and the positions reconstructed by the MWPC [i.e., histograms of $\left(x_{\mathrm{MWPC}}-x_{\text {nom }}\right)$ and $\left.\left(y_{\mathrm{MWPC}}-y_{\text {nom }}\right)\right]$. These difference distributions are shown for the detector 1 reconstruction in Fig. 19.

The dashed curves are the results of Gaussian fits to the distributions, with the means fitted as free parameters. Note that a slight mispositioning $(\sim$ few $\mathrm{mm}$ ) of the detectors relative to the magnetic field lines may contribute to the non-zero fitted means. Because we were interested in assessing the resolution relative to the nominal positions, we also performed constrainedGaussian fits to these distributions, in which the means were fixed to be 0; the results of these fits are shown as the solid curves. Because the differences between the fitted widths from the free- and constrained-Gaussian fits were not great, we defined the average of the two fitted widths to be the spectrometer position resolution. The resulting values are listed in Table 1. These data have demonstrated that the position resolution of the spectrometer is $\sim 2.0 \mathrm{~mm}$ over the fiducial volume. 

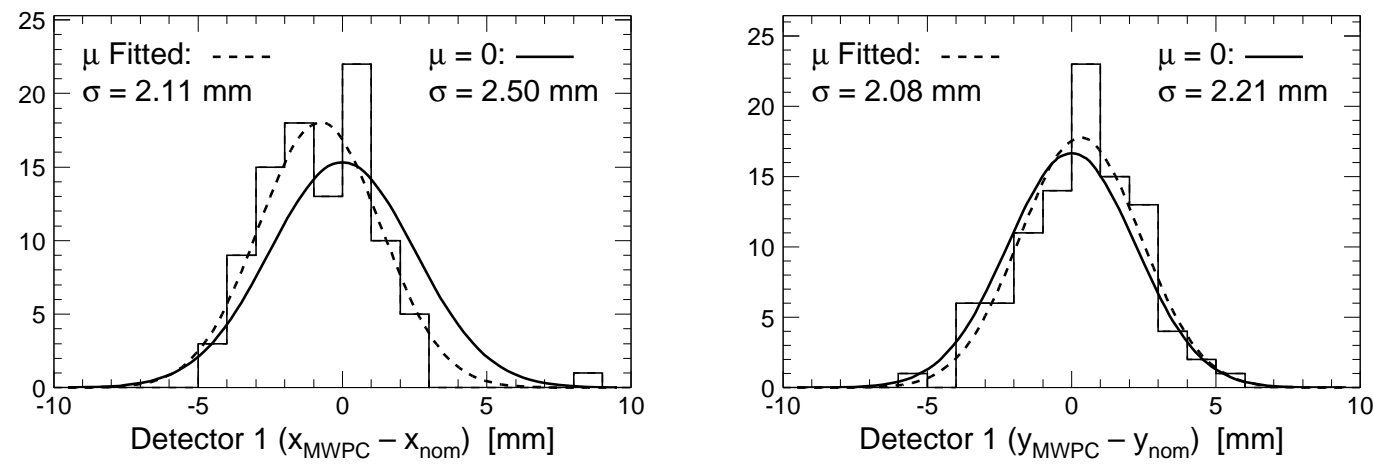

Fig. 19. Histograms of $\left(x_{\mathrm{MWPC}}-x_{\mathrm{nom}}\right)$ and $\left(y_{\mathrm{MWPC}}-y_{\text {nom }}\right)$ difference distributions for reconstruction by the dectector $1 \mathrm{MWPC}$. The dashed (solid) curves are the results of Gaussian fits in which the means were fitted as a free parameter (constrained to be 0$)$.

\section{Table 1}

Extracted values of the spectrometer position resolution from MWPC position reconstruction. Listed are the fitted widths for the free- and constrained-Gaussian fits to the reconstructed-nominal position difference distributions. The position resolution for each coordinate is defined to be the average of the free and constrained fitted widths.

\begin{tabular}{lccc}
\hline \hline & \multicolumn{2}{c}{ Fitted Widths $\sigma[\mathrm{mm}]$} & Position \\
Reconstruction & Free-Fit $\mu$ & Constrained $\mu=0$ & Resolution $[\mathrm{mm}]$ \\
\hline \hline Detector 1 MWPC $x$ & 2.11 & 2.50 & 2.31 \\
Detector 1 MWPC $y$ & 2.08 & 2.21 & 2.15 \\
\hline Detector 2 MWPC $x$ & 1.57 & 2.14 & 1.86 \\
Detector 2 MWPC $y$ & 1.55 & 1.45 & 1.50 \\
\hline \hline
\end{tabular}

\subsection{Spectrometer instrumental asymmetry}

We analyzed the data from all of our ${ }^{113} \mathrm{Sn}$ calibration runs and calculated an instrumental asymmetry, denoted $\xi$, for Type 0 events according to

$$
\xi=\frac{N_{1}-N_{2}}{N_{1}+N_{2}}
$$

We found that the mean instrumental asymmetry for those runs satisfying the fiducial volume radius cut of $41.5 \mathrm{~mm}$ is $\xi=(-0.400 \pm 0.083) \%$.

The instrumental asymmetry is itself $\sim 10 \%$ of the expected physics asymmetry, $A_{\exp }=P_{n} A\langle\beta \cos \theta\rangle \sim 4.5 \%$. The origin of a non-zero instrumental asymmetry (from an isotropic source) is likely the choice of cuts (e.g., cuts on 
the MWPC anode spectra, or cuts for muon identification) for the event selection. Although non-zero, the instrumental asymmetry cancels to all orders in the extraction of the $\beta$-asymmetry $A$ from the experimental super-ratio, Eq. (5), as the super-ratio asymmetry is insensitive to differences between the two detectors' efficiencies.

\section{Summary}

In this paper we described a solenoidal electron spectrometer designed for a precision measurement of the neutron $\beta$-asymmetry with ultracold neutrons. This spectrometer consists of a 1.0-Tesla superconducting solenoid, with a combined MWPC and plastic scintillator detector package for detection of the $\beta$-decay electrons. The UCNA experiment is currently operating at the Los Alamos National Laboratory, and collection of $\beta$-asymmetry data is ongoing. Further experiments to perform a precise measurement of the neutron $\beta$-decay energy spectrum, and to extract small energy-dependent terms in the $\beta$-asymmetry due to recoil-order corrections, are possible if the spectrometer's energy resolution can be improved. This will be achieved by implementing large-area silicon detectors as an alternate technology to the plastic scintillator detectors. Other experiments aimed at the determination of other angular correlation parameters, such as $a$ and $B$ in Eq. (1), can be conducted if proton detection can be incorporated into the apparatus. We intend to achieve proton detection by introducing thin secondary-electron-emitting foils into the apparatus, into which the decay protons would be accelerated by an electrostatic potential. Both initiatives in detector technology development are ongoing at this time.

We conclude this paper by emphasizing that our techniques and results for the rejection of gamma-ray induced events and an assessment of the spectrometer's position resolution are generally applicable to a variety of precision $\beta$-decay experiments utilizing neutrons and nuclei.

\section{Acknowledgments}

We thank R. Cortez and J. Pendlay for their skillful technical contributions to the design, fabrication, and deployment of the detector systems. We thank S. Currie for his devoted efforts to the operation and maintenance of the helium liquefaction plant. We thank the entire UCNA collaboration for many valuable suggestions. This work was supported in part by the National Science Foundation under grant numbers PHY-0079767 (a Major Research Instrumentation Program grant), PHY-0244899, PHY-0555674, and also by the Natural Sciences and Engineering Research Council of Canada. 


\section{References}

[1] J. S. Nico and W. M. Snow, Annu. Rev. Nucl. Part. Sci. 55, 27 (2005).

[2] N. Severijns and M. Beck, Rev. Mod. Phys. 78, 991 (2006).

[3] H. Abele, Prog. Part. Nucl. Phys. 60, 1 (2008).

[4] J. D. Jackson, S. B. Treiman, and H. W. Wyld, Jr., Phys. Rev. 106, 517 (1957).

[5] D. H. Wilkinson, Nucl. Phys. A377, 474 (1982).

[6] S. Gardner and C. Zhang, Phys. Rev. Lett. 86, 5666 (2001).

[7] A. Czarnecki, W. J. Marciano, and A. Sirlin, Phys. Rev. D 70, 093006 (2004); W. J. Marciano and A. Sirlin, Phys. Rev. Lett. 96, 032002 (2006).

[8] H. Abele et al., Eur. Phys. J. C 33, 1 (2004).

[9] J. C. Hardy and I. S. Towner, Phys. Rev. Lett. 94, 092502 (2005); J. C. Hardy and I. S. Towner, Phys. Rev. C 71, 055501 (2005).

[10] The UCNA experiment, A. Saunders and A. R. Young, spokespersons; R. Carr et al., "Technical review report for an accurate measurement of the neutron spin - electron angular correlation in polarized neutron beta decay with ultracold neutrons" (2000).

[11] H. Abele et al., Phys. Rev. Lett. 88, 211801 (2002).

[12] T. M. Ito et al., Nucl. Instrum. Methods Phys. Res. A 571, 676 (2007).

[13] C. L. Morris et al., Phys. Rev. Lett. 89, 272501 (2002).

[14] A. Saunders et al., Phys. Lett. B 593, 55 (2004).

[15] J. D. Jackson, Classical Electrodynamics, Third Edition (John Wiley \& Sons, Inc., New York, 1999).

[16] J. W. Martin et al., Phys. Rev. C 68, 055503 (2003); J. W. Martin et al., Phys. Rev. C 73, 015501 (2006).

[17] M. Schumann and H. Abele, Nucl. Instrum. Methods Phys. Res. A 585, 88 (2008).

[18] T. Tabata, R. Ito, and S. Okabe, Nucl. Instrum. Methods 94, 509 (1971).

[19] Manuscript in preparation.

[20] J. Yuan, Ph.D. thesis, California Institute of Technology (2006).

[21] American Magnetics, Inc., www.americanmagnetics.com.

[22] Meyer Tool and Manufacturing, Inc., www.mtm-inc.com.

[23] Eljen Technology, www.eljentechnology.com. 
[24] Suzuno Giken, http://www .apace-science.com/tie_up/suzuno.htm.

[25] Burle Technologies, Inc., www . burle.com/cgi-bin/byteserver.pl/pdf/8850.pdf.

[26] MIDAS Data Acquisition System, midas.psi.ch or midas.triumf.ca. 